\title{
AIP
}

\section{Structure and short-time dynamics in concentrated suspensions of charged colloids}

Fabian Westermeier, Birgit Fischer, Wojciech Roseker, Gerhard Grübel, Gerhard Nägele et al.

Citation: J. Chem. Phys. 137, 114504 (2012); doi: 10.1063/1.4751544

View online: http://dx.doi.org/10.1063/1.4751544

View Table of Contents: http://jcp.aip.org/resource/1/JCPSA6/v137/i11

Published by the American Institute of Physics.

\section{Additional information on J. Chem. Phys.}

Journal Homepage: http://jcp.aip.org/

Journal Information: http://jcp.aip.org/about/about_the_journal

Top downloads: http://jcp.aip.org/features/most_downloaded

Information for Authors: http://jcp.aip.org/authors

\section{ADVERTISEMENT}

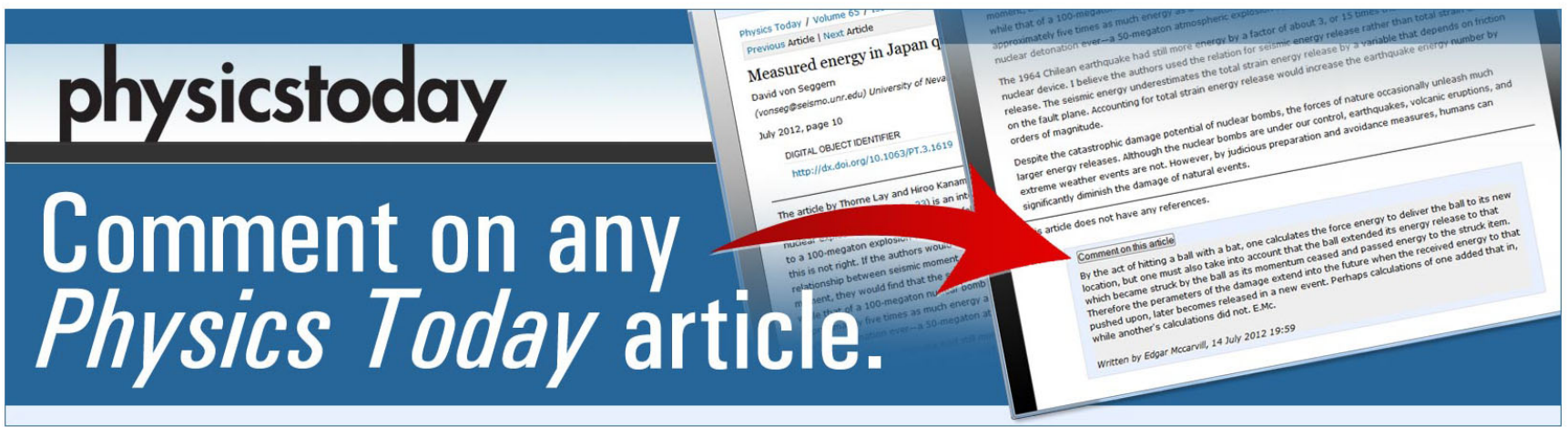




\title{
Structure and short-time dynamics in concentrated suspensions of charged colloids
}

\author{
Fabian Westermeier, ${ }^{1, a)}$ Birgit Fischer, ${ }^{1}$ Wojciech Roseker, ${ }^{1}$ Gerhard Grübel, ${ }^{1}$ \\ Gerhard Nägele, ${ }^{2}$ and Marco Heinen $\left.{ }^{3, b}\right)$ \\ ${ }^{1}$ Hasylab, Deutsches Elektronen-Synchrotron, Notkestrasse 85, D-22607 Hamburg, Germany \\ ${ }^{2}$ Institute of Complex Systems (ICS-3), Forschungszentrum Jülich, D-52425 Jülich, Germany \\ ${ }^{3}$ Institut für Theoretische Physik II, Weiche Materie, Heinrich-Heine-Universität Düsseldorf, \\ D-40225 Düsseldorf, Germany
}

(Received 5 May 2012; accepted 27 August 2012; published online 18 September 2012)

\begin{abstract}
We report a comprehensive joint experimental-theoretical study of the equilibrium pair-structure and short-time diffusion in aqueous suspensions of highly charged poly-acrylate (PA) spheres in the colloidal fluid phase. Low-polydispersity PA sphere systems with two different hard-core radii, $R_{0}=542$ and $1117 \AA$, are explored over a wide range of concentrations and salinities using static and dynamic light scattering (DLS), small angle x-ray scattering, and x-ray photon correlation spectroscopy (XPCS). The measured static and dynamic scattering functions are analyzed using stateof-the-art theoretical methods. For all samples, the measured static structure factor, $S(Q)$, is in good agreement with results by an analytical integral equation method for particles interacting by a repulsive screened Coulomb plus hard-core pair potential. In our DLS and XPCS measurements, we have determined the short-time diffusion function $D(Q)=D_{0} H(Q) / S(Q)$, comprising the free diffusion coefficient $D_{0}$ and the hydrodynamic function $H(Q)$. The latter is calculated analytically using a selfpart corrected version of the $\delta \gamma$-scheme by Beenakker and Mazur which accounts approximately for many-body hydrodynamic interactions (HIs). Except for low-salinity systems at the highest investigated volume fraction $\phi \approx 0.32$, the theoretical predictions for $H(Q)$ are in excellent agreement with the experimental data. In particular, the increase in the collective diffusion coefficient $D_{c}=D(Q$ $\rightarrow 0$ ), and the decrease of the self-diffusion coefficient, $D_{s}=D(Q \rightarrow \infty)$, with increasing $\phi$ is well described. In accord with the theoretical prediction, the peak value, $H\left(Q_{m}\right)$, of $H(Q)$ relates to the nearest neighbor cage size $\sim 2 \pi / Q_{m}$, for which concentration scaling relations are discussed. The peak values $H\left(Q_{m}\right)$ are globally bound from below by the corresponding neutral hard-spheres peak values, and from above by the limiting peak values for low-salinity charge-stabilized systems. HIs usually slow short-time diffusion on colloidal length scales, except for the cage diffusion coefficient, $D_{\text {cge }}=D\left(Q_{m}\right)$, in dilute low-salinity systems where a speed up of the system dynamics and corresponding peak values of $H\left(Q_{m}\right)>1$ are observed experimentally and theoretically. () 2012 American Institute of Physics. [http://dx.doi.org/10.1063/1.4751544]
\end{abstract}

\section{INTRODUCTION}

The existence of a large variety of well-characterized colloidal model systems has triggered extensive research on the dynamics of colloidal Brownian particles over the past decades. ${ }^{1-5}$ This has led to a developed understanding of the microstructure and the dynamics in fluid-like colloidal suspensions. In the present paper, we report on our comprehensive study of aqueous suspensions of charge-stabilized poly-acrylate (PA) spherical particles, which combines stateof-the-art experimental with recently developed theoretical methods. We have synthesized and investigated a large number of PA samples with different particle diameters and size polydispersities, in a wide range of colloid volume fractions and electrolyte concentrations, covering practically the complete fluid-phase state. For the here discussed PA spheres with mean radii of 542 and $1117 \AA$, the relevant length scales

a) fabian.westermeier@desy.de.

b) mheinen@thphy.uni-duesseldorf.de. of the structural and dynamical features can be conveniently accessed by static and dynamic light scattering (SLS/DLS), provided that the samples are sufficiently dilute to avoid multiple scattering. For the PA systems at higher concentrations, where for visible light multiple scattering becomes nonnegligible, we have applied static and dynamic x-ray scattering techniques using a partially coherent, third-generation synchrotron source. ${ }^{6-8}$ These techniques have the advantage of covering an extended scattering wavevector range. We have analyzed our experimental results using the recently introduced modified penetrating background-corrected rescaled mean spherical approximation (MPB-RMSA) ${ }^{9,10}$ method of calculating the static pair-correlations, and using the self-part corrected $\delta \gamma$-scheme ${ }^{11}$ for the short-time dynamics of concentrated charge-stabilized colloidal suspensions.

Both the strength and the range of the direct (i.e., nonhydrodynamic) interactions in charge-stabilized colloidal systems are experimentally adjustable by varying the colloid volume fraction, $\phi$, and the concentration of added salt-ion pairs, $n_{s}(\mathrm{KCl}$ in our case). The colloidal particles have a steric 
excluded volume plus an exponentially screened electrostatic repulsion, with the Debye screening length decreasing with increasing $\phi$ and $n_{s}$. At low particle concentrations and salinities, the direct interactions are long-ranged, and near-contact configurations of colloidal spheres are suppressed. At high salinity, the electrostatic repulsion is fully screened. On neglecting short-range van der Waals (vdW) attraction, the pair potential reduces then to the hard-core potential describing neutral hard-spheres.

In addition to direct electro-steric interactions, the dynamics of colloidal particles is influenced also by hydrodynamic interactions (HIs) that couple the motions of the colloids through the low Reynolds-number flow of the suspending viscous solvent. An accurate inclusion of HIs into a theoretical scheme of (short-time) diffusion in concentrated systems is a non-trivial task, due to the many-body character of the HIs. As an additional complication for the theoretical treatment, HIs are the longest ranged particle interactions, decaying asymptotically with the inverse inter-particle distance.

In suspensions of neutral colloidal hard spheres, HIs slow the short-time diffusion on all colloidal length scales by an amount that increases with increasing $\phi$. For charged colloidal systems on the other hand, the interplay of electrostatic repulsion and HIs gives rise to an intricate concentrationscaling behavior of various diffusion coefficients. For example, charge-stabilized colloidal suspensions at low salinity have a collective diffusion coefficient, $D_{c}$, that varies nonmonotonically as a function of $\phi \cdot{ }^{12-16}$ Moreover, the collective diffusive motion on the length scale of the nearest neighbor cage diameter, as described by $D\left(Q_{m}\right)$, is enhanced rather than slowed hydrodynamically at low-salt and low- $\phi$ conditions. ${ }^{11,17-25}$ The hydrodynamic effects described here are analyzed in the present work both experimentally and theoretically.

The static structure factor, $S(Q)$, and the hydrodynamic function, $H(Q)$, predicted by our analytic theoretical methods are shown to be in excellent agreement with our visible lightand $\mathrm{x}$-ray scattering results for all investigated salt concentrations, and for all probed colloidal volume fractions up to $\phi$ $=0.16$. We demonstrate that the predictions of the self-part corrected $\delta \gamma$-scheme for $H(Q)$ and $D(Q)$ become less accurate for low-salinity suspensions at a large volume fraction of $\phi=0.32$, indicating that a more refined analytical theoretical scheme needs to be developed which covers also this highconcentration regime.

The paper is organized as follows: In Sec. II, we describe our sample preparation, and our static and dynamic visible light- and x-ray scattering experiments. In Sec. III, the essentials of the theory of static and dynamic scattering are presented, with focus on the underlying hard-sphere plus repulsive Yukawa (HSY) pair potential model, and the employed theoretical methods for the statics and short-time dynamics. Section IV includes our experimental and theoretical results on the static scattering functions of the PA sphere systems, and a discussion of the limiting concentration scaling relations fulfilled by the nearest neighbor cage radius. Section V compares our short-time dynamics experimental scattering data to the corresponding theoretical predictions. It includes also a discussion of the limiting concentration scaling of the various diffusion coefficients of charged and of effectively neutral colloidal spheres. Our conclusions are presented in Sec. VI. An index of abbreviations and symbols is given at the end of the paper.

\section{EXPERIMENTAL DETAILS}

\section{A. Sample preparation}

By means of radical emulsion polymerization, ${ }^{26}$ we have synthesized two batches of PA spherical colloidal particles with two different mean hard-core radii, $R_{0}=542$ and $1117 \AA$ As. As explained in detail in Subsection IV A, we have determined these mean radii, and the corresponding sizepolydispersities, from model fits to the small angle x-ray scattering (SAXS) data. In the following, we refer to the two batches as the $\mathrm{PA}_{542}$ and $\mathrm{PA}_{1117}$ systems.

To enhance the electron density and hence the scattering strength of the polymer particles, two different functionalized monomers containing either fluorine or silicon atoms $(1 \mathrm{H}, 1 \mathrm{H}, 5 \mathrm{H}$-octafluoropentylmethacrylate (ABCR $\mathrm{GmbH} \&$ Co. KG, Germany) and methacryloxymethyltrimethylsilane (ABCR GmbH \& Co. KG, Germany)) were used for the radical emulsion polymerization. The structural formulae of the monomers are displayed in Fig. 1, the syntheses were performed as described by Härtl. ${ }^{27}$ The reaction temperature of $57.5^{\circ} \mathrm{C}$ was kept constant during the synthesis. A mixture with a 1:1 molar ratio of the two monomers was added to a water amount of $1.5 l$, saturated with nitrogen to remove dissolved oxygen. In addition, the cross-linker 2,2,3,3,4,4,5,5octafluorohexanediol-1,6-dimethacrylate (ABCR GmbH \& Co. KG, Germany) was added to stabilize the formed nanoparticles. As a redox-system, a solution of $\mathrm{NaSO}_{3}$ (Fluka, Switzerland) and $\left(\mathrm{NH}_{4}\right)_{2} \mathrm{Fe}\left(\mathrm{SO}_{4}\right)_{2}$ (Fluka, Switzerland) in deionized water was added. The polymerization was initialized by adding a solution of the initiator $\mathrm{K}_{2} \mathrm{~S}_{2} \mathrm{O}_{8}$ (Merck, Germany) in deionised water.

For the $\mathrm{PA}_{1117}$ system, a subsequent synthesis step was carried out at identical synthesis conditions $24 \mathrm{~h}$ after the first synthesis step, resulting in a further growth of the particles. At the end of the synthesis, remnant monomer and lowmolecular-weight oligomers were removed by a 10-12 days dialysis against deionized water. The purified colloidal suspension was concentrated by vacuum distillation. Remaining electrolyte ions were removed by a mixed-bed ion exchanger (Merck, Germany).

In the experiments, the ion exchanger was removed, and the concentration of colloidal particles was adjusted either by dilution with deionised water, or by a gentle centrifugation over a $24 \mathrm{~h}$ period, resulting in the latter case in a
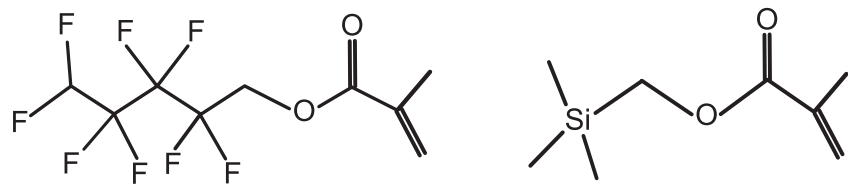

FIG. 1. Structural formulae of the two monomers used for the radical emulsion polymerization. Left: $1 \mathrm{H}, 1 \mathrm{H}, 5 \mathrm{H}$-octafluoropentylmethacrylate. Right: methacryloxymethyltrimethylsilane. 
soft sediment of colloidal particles which was easily redispersed. To obtain systems with added electrolyte, a small volume of differently concentrated dissolved potassium chloride ( $\mathrm{KCl}$, Merck, Germany) in deionized water was added in replacement of the same volume of deionized water. The poly-acrylate systems studied here exhibit a high effective particle charge due to the deprotonation of surface sulfate groups.

\section{B. Visible light scattering}

The SLS and DLS experiments were performed using a 3D-DLS Spectrometer spectrometer-goniometer-system (LS Instruments AG, Switzerland), operated in multiple-scattering suppressing cross-correlation mode. This mode involves splitting the incident laser beam of wavelength $\lambda=632.8 \mathrm{~nm}$ into two beams of equal intensity by a prism system. Both beams are focused by a lens on the same scattering volume in the sample cell. The scattered light is defocused by another lens, and detected by two avalanche photo diodes placed on a goniometer arm which allows to detect the scattered light at scattering angles in between $30^{\circ}$ and $150^{\circ}$.

\section{X-ray scattering}

Our x-ray scattering experiments have been performed at the beamline ID10A of the European Synchrotron Radiation Facility in Grenoble. ${ }^{7,28}$ The x-ray beam was monochromatized to a photon energy of $7.98 \mathrm{keV}$, corresponding to a wavelength $\lambda$ of $1.554 \AA$. The monochromator bandwidth, $\Delta \lambda / \lambda$ $\approx 1.4 \times 10^{-4}$, corresponds to a longitudinal coherence length of about $1 \mu \mathrm{m}$. The x-ray beam was subsequently collimated to a final width by a set of beam-defining slits, resulting in a beam area of typically in between $10 \mu \mathrm{m} \times 10 \mu \mathrm{m}$ and $20 \mu \mathrm{m} \times 20 \mu \mathrm{m}$.

The Fraunhofer fringes resulting from the beam defining slits, and additional stray scattering, have been suppressed by a tantalum guard slit placed directly in front of the sample. The scattered intensity has been detected by a two-dimensional (2D) detector in case of time-averaging experiments, and by a point detector in the case of photon correlation experiments. Both detectors were interchangeable during the experiment, enabling thus the measurement of the static and dynamic properties for the same sample. The detectors were placed at a detector-to-sample distance of $2.2 \mathrm{~m}$.

Our usage of a 2D detector gives enhanced statistics for the time-averaged scattering experiments, especially at high values of the scattering wavenumber $Q$, where the count rate is drastically decreasing due to the $Q^{-4}$ dependence of the scattered intensity of spherical colloidal particles. The employed 2D detector was a charge-coupled device detector with a total area of $1242 \times 1152$ pixels and a pixel size of $22.5 \mu \mathrm{m}$ $\times 22.5 \mu \mathrm{m}$. Typically, series of 100-200 frames with an exposure time of $0.1 \mathrm{~s}-10 \mathrm{~s}$ were taken, subsequently averaged, and corrected for detector background. The time-averaged data were normalized relative to the incident flux, provided by a monitor detector, and to the transmission of the sample.
We have recorded SAXS spectra of the pure solvent and subtracted them from the data of the colloidal dispersions. The time-averaged scattering pattern from the fluid-like colloidal samples studied here are completely isotropic. Thus, in order to achieve better statistics, we have azimuthally averaged the recorded $2 \mathrm{D}$ scattering patterns.

Since the dynamics of the probed colloidal particles is exceedingly faster than the time-resolution of present-day $2 \mathrm{D}$ $\mathrm{X}$-ray detectors, an avalanche photo diode was used instead for the x-ray photon correlation spectroscopy (XPCS) measurements. Slits directly in front of the point detector were adjusted to a size in between $50 \mu \mathrm{m} \times 50 \mu \mathrm{m}$ and $100 \mu \mathrm{m}$ $\times 100 \mu \mathrm{m}$.

To prevent x-ray beam damage of the samples, a flow device was used where the sample was pumped through a quartz capillary with a diameter of $1 \mathrm{~mm}$. To pump the sample, a syringe pump was used allowing for constant small flow-rates in the $\mu \mathrm{l} / \mathrm{h}$ range. The travel time of colloidal particles in the beam was varied between $1 \mathrm{~s}$ and $0.1 \mathrm{~s}$. In addition to preventing beam-damage, the flow device allows for a precise measurement and a subsequent subtraction of the scattering intensity due to the solvent and the capillary.

\section{THEORY}

\section{A. Static scattering function}

The time-averaged intensity, $I_{s}$, of x-rays or visible light scattered by the colloidal particles into a solid angle, $\Delta \Omega$, is given by ${ }^{29,30}$

$$
I_{s}=\frac{\mathrm{d} \sigma}{\mathrm{d} \Omega} \frac{I_{0}}{A_{0}} \Delta \Omega,
$$

where $I_{0}$ is the intensity of the incident beam of crosssectional area $A_{0}$, and $\mathrm{d} \sigma / \mathrm{d} \Omega$ is the differential scattering cross-section. In the absence of multiple scattering, the crosssection of a homogeneous fluid-like sample of spherical particles can be approximated by ${ }^{29,30}$

$$
\frac{\mathrm{d} \sigma}{\mathrm{d} \Omega}=n V_{s} F_{s}^{2} P_{m}(Q) S_{m}(Q),
$$

where $n$ is the number density of colloidal particles, and $V_{s}$ is the scattering volume. The scattering amplitude, $F_{s}$, is different for the X-ray and visible light scattering cases. For x-ray scattering, $F_{s}=r_{0} \Delta \rho$, where $r_{0}$ is the electronic scattering length, and $\Delta \rho=\rho_{c}-\rho_{s}$ is the contrast between the electron densities, $\rho_{c}$ and $\rho_{s}$, of scatterers and solvent, respectively. For visible light scattering and optically homogeneously scattering colloids, $F_{s}=\left(\epsilon_{r}-\epsilon_{s}\right) / \epsilon_{s}$ is given by the dielectric contrast, with $\epsilon_{r}$ and $\epsilon_{s}$ denoting the colloid and solvent dielectric optical permittivities at the employed laser-light frequency. In Eq. (2), $P_{m}(Q)$ is the size-averaged colloidal form factor, and $S_{m}(Q)$ is the measurable colloidal static structure factor which, for polydisperse samples, is a weighted sum of partial static structure factors. ${ }^{14,31}$

The mean form factor of size-polydisperse spherical particles with size distribution function $p(R)$ is given by ${ }^{32}$

$$
P_{m}(Q)=\frac{\int_{0}^{\infty} \mathrm{d} R p(R) f_{\mathrm{sph}}^{2}(Q R) V_{\mathrm{sph}}^{2}}{\int_{0}^{\infty} \mathrm{d} R p(R) V_{\mathrm{sph}}^{2}} .
$$


Here, $f_{\mathrm{sph}}(Q R)=3[\sin (Q R)-Q R \cos (Q R)] /(Q R)^{3}$ and $V_{\mathrm{sph}}=(4 / 3) \pi R^{3}$ are the form amplitude and the volume of a colloidal sphere of radius $R$, respectively.

By invoking the so-called decoupling approximation, we neglect the small size-, and thus putatively also small chargepolydispersity effects on the colloidal microstructure, while maintaining the polydispersity influence on the scattering amplitudes. The decoupling approximation ${ }^{14}$ consists of approximating the exact $S_{m}(Q)$ in Eq. (2) by

$$
S_{m}^{d}(Q)=[1-X(Q)]+X(Q) S(Q),
$$

where $S(Q)$ is the structure factor of ideally monodisperse spheres of radius equal to the mean radius $R_{0}=\langle R\rangle$. The decoupling amplitude factor $X(Q)$, with $0 \leq X(Q) \leq 1$ is given by

$$
X(Q)=\frac{\left[\int_{0}^{\infty} \mathrm{d} R p(R) f_{\mathrm{sph}}(Q R) V_{\mathrm{sph}}\right]^{2}}{P_{m}(Q) \int_{0}^{\infty} \mathrm{d} R p(R) V_{\mathrm{sph}}^{2}} .
$$

The function $1-X(Q)$ is displayed in Fig. 2 for the systems $\mathrm{PA}_{542}$ and $\mathrm{PA}_{1117}$, along with the corresponding $P_{m}(Q)$. For moderately small size-polydispersity, $X\left(Q R_{0} \lesssim 3 / 4\right) \approx 1$ $-9\left(\Delta R / R_{0}\right)^{2},{ }^{14}$ as reflected by the low- $Q$ plateau of $1-X(Q)$ visible in Fig. 2. For larger values of $Q$, the decoupling amplitude exhibits oscillations, and it vanishes for $Q \rightarrow \infty$. Hence, the effects of polydispersity as described in the decoupling approximation are oscillations of $S_{m}^{d}(Q)$ which are less pronounced as those of $S(Q)$, and the appearance of an incoherent scattering contribution at low $Q$ given by $[1-X(Q)]$. A proper account of the incoherent low $Q$ scattering contribution is important for strongly repelling charged colloidal particles characterized by a small reduced isothermal osmotic com-

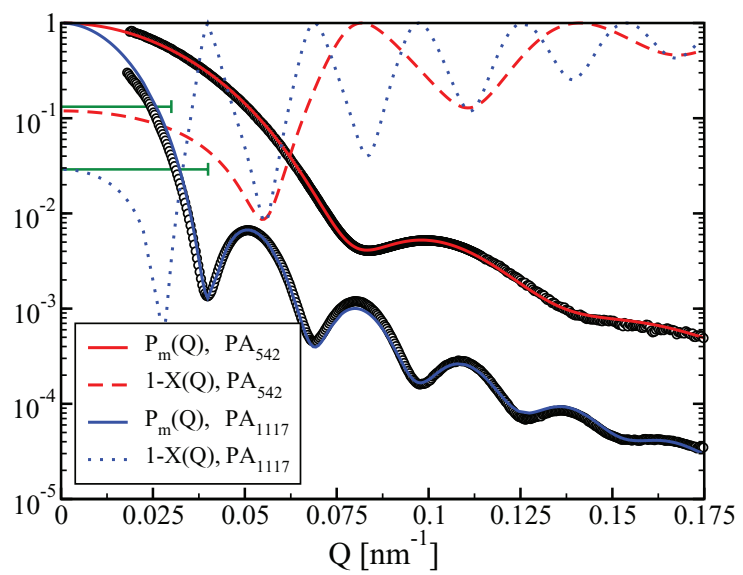

FIG. 2. Black open circles: Normalized SAXS intensities (measurable form factors) for dilute samples $\mathrm{PA}_{542}$ (upper curve) and $\mathrm{PA}_{1117}$ (lower curve), at high salt concentrations of $n_{s}=200 \mu \mathrm{M}$ for $\mathrm{PA}_{542}$, and $n_{s}=500 \mu \mathrm{M}$ for $\mathrm{PA}_{1117}$, and a common volume fraction $\phi<0.005$. Solid red and blue curves: Best fit form factor, $P_{m}(Q)$, according to Eq. (3), for the unimodal SchulzZimm size distribution given in Eq. (6). From the fit, mean particle radii $R_{0}$ $=542$ and $1117 \AA$ and polydispersities $\Delta R / R_{0}=0.121$ and 0.057 have been deduced for systems $\mathrm{PA}_{542}$ and $\mathrm{PA}_{1117}$, respectively. Dashed red and dotted blue curves: Factor $1-X(Q)$ of systems $\mathrm{PA}_{542}$ and $\mathrm{PA}_{1117}$, respectively, with the decoupling amplitude, $X(Q)$, according to Eq. (5). The horizontal green line segments span the $Q$-intervals for which $H(Q)$ is shown in Figs. 6 and 7. Note that $1-X(Q \rightarrow 0) \approx 9\left(\Delta R / R_{0}\right)^{2}=0.132$ and 0.029 for systems $\mathrm{PA}_{542}$ and $\mathrm{PA}_{1117}$, respectively. pressibility $S(Q \rightarrow 0) \approx 0$, since in this case it dominates the coherent scattering contribution $X(Q) S(Q)$.

To describe the size polydispersity in our theoretical analysis, we use the two-parametric, unimodal Schulz-Zimm ${ }^{33,34}$ distribution function,

$$
p(R)=\frac{R^{t}}{\Gamma(t+1)}\left(\frac{t+1}{R_{0}}\right)^{t+1} \exp \left(-\frac{t+1}{R_{0}} R\right),
$$

involving the Gamma function $\Gamma$, and polydispersity (relative standard deviation) $\Delta R / R_{0}=R_{0}^{-1}\left[\left\langle R^{2}\right\rangle-\langle R\rangle^{2}\right]^{1 / 2}=[1 /(t$ $+1)]^{1 / 2}$. We have determined the mean radius $R_{0}$ and polydispersity $\Delta R / R_{0}$ for systems $\mathrm{PA}_{542}$ and $\mathrm{PA}_{1117}$ by fitting $P_{m}(Q)$ to the normalized SAXS intensities of strongly dilute, highsalinity systems where $S_{m}(Q)$ is practically identical to one. The resulting fit values are discussed in Subsection IV A, in context with Fig. 2.

According to Eqs. (2) and (4), evaluation of the scattering cross-section requires the computation of $S_{m}(Q) \approx S_{m}^{d}(Q)$ via $X(Q)$ and $S(Q)$. In calculating $S(Q)$, we assume the particles to interact directly by the repulsive part,

$$
\beta u(x)= \begin{cases}\infty, & x=r /\left(2 R_{0}\right) \leq 1, \\ \gamma \frac{e^{-k x}}{x}, & x>1,\end{cases}
$$

of the Derjaguin-Landau-Verwey-Overbeek (DLVO) pair potential, ${ }^{35}$ characterized by the pair interaction amplitude, $\gamma$, and the reduced screening parameter, $k$, where

$$
\gamma=\frac{L_{B}}{2 R_{0}}\left(\frac{Z e^{k / 2}}{1+k / 2}\right)^{2}
$$

and

$$
k^{2}=\frac{L_{B} / R_{0}}{1-\phi}\left(12 \phi|Z|+4 \pi n_{s}\left(2 R_{0}\right)^{3}\right) .
$$

Here, $\beta=1 / k_{B} T$, with Boltzmann constant $k_{B}$ and absolute temperature $\mathrm{T}$, and $L_{B}=\beta e^{2} / \epsilon$ is the solvent-characteristic Bjerrum length in Gaussian units with proton elementary charge $e$ and zero-frequency limiting dielectric constant, $\epsilon$, of the solvent. The parameters $\gamma$ and $k$ are determined by the number density, $n_{s}$, of 1-1 salt co-ions, the colloid volume fraction $\phi=(4 \pi / 3) n R_{0}^{3}$, involving the number density, $n$, of colloidal particles, and the effective colloid charge number, $Z$. For weakly charged colloids, $Z$ is equal to the colloidal bare charge, i.e., the number of dissociated monovalent ionic surface groups on a colloidal particle. For the highly charged colloids in the present study, where $L_{B} Z /\left(2 R_{0}\right) \gtrsim 1$, one has to regard $Z$ as the net charge of a colloid dressed with closely associated counterions. ${ }^{36-46}$ The factor $1 /(1-\phi)$ in Eq. (9) corrects for the free volume accessible to point-like microions at nonzero concentrations of impermeable colloidal spheres. ${ }^{47,48}$

We note again that in the framework of the employed decoupling approximation, size polydispersity and a possibly related particle charge polydispersity are ignored in the employed pair potential. According to charge-renormalization theory predictions in the saturation regime of highly charged colloidal particles, the effective particle charge is only weakly (i.e., logarithmically) dependent on the bare colloidal charge and particle radius. ${ }^{43-46}$ 
Furthermore, in using the pair potential according to Eq. (7), referred to as the hard-sphere plus repulsive Yukawa potential, attractive interaction contributions such as vdW attraction are neglected. For highly charged particles at low salinity, the DLVO potential with vdW attraction included has a pronounced principal maximum (Coulomb barrier) where $\beta u(x \gtrsim 1) \gg 1$, so that close-contact pair configurations are extremely unlikely. In addition to low-salinity samples, we have studied colloidal suspensions with a large amount of added salt. Despite a nearly vanishing Coulomb barrier, also these samples show no sign of precipitation. The stability of these high-salinity systems might be due to the hydrophilic character of the highly charged PA spheres, and a rather weak strength of the vdW attraction due to surface roughness. This justifies to some extent our neglect of short-range attraction and size polydispersity in using Eq. (7) for not too large concentrations, with further credibility of their neglect drawn from the good and consistent fits of the SLS- and SAXSdetermined $S_{m}(Q)$ by our theoretical results based on the HSY pair potential in Eq. (7) (cf., Subsection IV B).

To calculate $S(Q)$ using the pair potential in Eq. (7), we employ the MPB-RMSA, an analytic method recently introduced by two of the present authors. ${ }^{9,10}$ It is based on Snook and Hayter's modification ${ }^{49}$ of the well-known RMSA scheme. ${ }^{50}$ The details of the MPB-RMSA method are given in Ref. 9, where also comprehensive parameter scans are described which cover the full fluid-state regime for chargestabilized particles interacting according to Eq. (7). The MPB-RMSA results for $S(Q)$ are generally in excellent agreement with far more elaborate Monte Carlo simulations, and with results by the well-established, but non-analytic RogersYoung integral equation scheme. ${ }^{51}$ For neutral particles, the MPB-RMSA solution for $S(Q)$ reduces to that obtained in the Percus-Yevick ${ }^{52,53}$ approximation.

\section{B. Dynamic scattering function}

In our DLS and XPCS experiments, we have determined the normalized intensity autocorrelation function, ${ }^{54}$

$$
g_{2}(Q, \tau)=\frac{\langle I(Q, t) I(Q, t+\tau)\rangle}{\langle I(Q)\rangle^{2}}=\beta(Q) g_{1}^{2}(Q, \tau)+1,
$$

as a function of wavenumber $Q$ and correlation time $\tau$. Here, $\beta(Q)$ denotes the speckle contrast, and $g_{1}(Q, \tau)$ is the normalized field autocorrelation function related to $g_{2}(Q, \tau)$ by the Siegert relation. The bracket, $\langle\ldots\rangle$, denotes a time average. For the fluid-disordered, isotropically scattering particle systems considered here, the average can be likewise interpreted as an equilibrium ensemble average. In the dynamical extension of the decoupling approximation, ${ }^{14}$

$$
g_{1}(Q, \tau)=[1-X(Q)] G(Q, \tau)+X(Q) S(Q, \tau),
$$

where $S(Q, \tau)$ is the intermediate scattering function, ${ }^{55}$ with $S(Q, \tau=0)=S(Q)$, and $G(Q, \tau)$ is the self-intermediate scattering function related to self-diffusion. For negligible nonGaussian contributions to $G(Q, \tau),{ }^{56}$ the self-intermediate scattering function is equal to $G(Q, \tau)=\exp \left\{-Q^{2} W(\tau)\right\}$, where $W(\tau)=1 / 6\left\langle|\mathbf{R}(0)-\mathbf{R}(\tau)|^{2}\right\rangle$ is the mean squared displacement (divided by the factor 6 in three-dimensions) of a sphere with center position $\mathbf{R}(\tau)$ at time $\tau$.

The colloidal short-time regime $\tau_{B} \approx \tau_{H} \ll \tau \ll \tau_{I}$ of the correlation time $\tau$ is constricted from below by the momentum relaxation time, $\tau_{B}=m /\left(6 \pi \eta_{0} R_{0}\right)$, roughly equal to the hydrodynamic vorticity diffusion time, $\tau_{H}=R_{0}^{2} \rho_{S} / \eta_{0}$, for overdamped shear waves diffusing across the radius $R_{0}$, and from above by the interaction time $\tau_{I}=4 R_{0}^{2} / D_{0} \cdot{ }^{14,57}$ Here, $m$ is the mass of a colloidal sphere, and $D_{0}=k_{B} T /\left(6 \pi \eta_{0} R_{0}\right)$ is the translational free diffusion coefficient of an isolated single sphere with hydrodynamic stick (i.e., no-slip) surface boundary conditions suspended in an infinite and quiescent (i.e., overall stationary) fluid. Moreover, $\rho_{S}$ and $\eta_{0}$ are the mass density and shear viscosity of the suspending Newtonian solvent. Note that our particles are practically neutrally buoyant. Using the viscosity, $\eta_{0}$, of water at room temperature, i.e., at $T=20^{\circ} \mathrm{C}$, for the systems $\mathrm{PA}_{542}$ and $\mathrm{PA}_{1117}$ we obtain $\tau_{B}$ $=0.7$ and $2.8 \mathrm{~ns}, \tau_{H}=2.9$ and $12 \mathrm{~ns}$, and $\tau_{I}=3.0$ and $26 \mathrm{~ms}$, respectively. During a short-time interval, $\tau$, a particle diffuses a distance which is only a tiny fraction of its size. This fact allows for calculating short-time diffusion properties from equilibrium averages involving Stokes-flow hydrodynamic mobilities.

In the colloidal short-time regime, $W(\tau) \approx D_{s} \tau$, where $D_{s}$ with $D_{s}<D_{0}$ is the short-time translational self-diffusion coefficient of a representative particle in a quiescent suspension of directly and hydrodynamically interacting particles. Furthermore, $S(Q, \tau) / S(Q) \approx \exp \left\{-Q^{2} D(Q) \tau\right\}$ in the colloidal short-time regime, with the short-time diffusion function $D(Q)=D_{0} H(Q) / S(Q)$. The positive-valued hydrodynamic function, $H(Q)$, describes the influence of HIs on shorttime diffusion. For the (hypothetical) case of hydrodynamically non-interacting particles, $H(Q) \equiv 1$. A value of $H(Q)$ smaller than one indicates a slowing influence of HIs on the short-time diffusion on a length scale $\sim 2 \pi / Q$, whereas $H(Q)$ $>1$ indicates a hydrodynamic diffusion enhancement.

The hydrodynamic function can be interpreted as the reduced short-time generalized mean sedimentation velocity of particles in a homogeneous suspension subject to a weak force field collinear with the scattering wavevector $\mathbf{Q}$ and oscillating spatially as $\cos (\mathbf{Q} \cdot \mathbf{r})$. Accordingly, $H(Q \rightarrow 0)$ is the reduced short-time sedimentation velocity (in the zero-volume flux reference frame) in a homogeneous force field in units of the corresponding sedimentation velocity of a single colloidal sphere. The related collective diffusion coefficient, $D_{c}$ $=D_{0} H(Q \rightarrow 0) / S(Q \rightarrow 0)$, quantifies the initial decay rate of thermally induced density waves of macroscopic wavelength. In the opposite limit of large wavenumbers, $H(Q \rightarrow \infty)$ $=D_{s} / D_{0}<1$. Note that $1 / S(Q \rightarrow 0)$ is commonly referred to as the thermodynamic factor associated with collective diffusion since, for monodisperse spherical particles, $S(0)$ is related to the isothermal osmotic compressibility. ${ }^{14}$

The shape of $H(Q)$ is quite similar to that of $S(Q)$, however, with less pronounced oscillations. In particular, the principal peaks of $H(Q)$ and $S(Q)$ are located at practically the same wavenumber, $Q_{m}$, inversely proportional to the diameter of the nearest neighbor cage formed around a colloidal particle. Therefore, $D_{\text {cge }}=D_{0} H\left(Q_{m}\right) / S\left(Q_{m}\right)$ is referred to as the cage-diffusion coefficient. While $H\left(Q_{m}\right)<1$ for many 
systems, in Sec. V we show that values $H\left(Q_{m}\right)>1$ are attained for suspensions at low salinity and low volume fraction.

In decoupling approximation at short times,

$$
g_{1}(Q, \tau)=[1-X(Q)] e^{-Q^{2} D_{s} \tau}+X(Q) S(Q) e^{-Q^{2} D(Q) \tau},
$$

with two exponentially decaying diffusive modes of relaxation rates determined by $D_{s}$ and $D(Q)$, respectively.

A first cumulant analysis of $g_{1}(Q, \tau)$ provides the measurable diffusion function,

$$
D_{m}(Q)=-\lim _{\tau \rightarrow 0} \frac{1}{Q^{2}} \frac{\partial}{\partial \tau} \ln g_{1}(Q, \tau)=D_{0} \frac{H_{m}(Q)}{S_{m}(Q)},
$$

which incorporates the measurable hydrodynamic function $H_{m}(Q)$. In decoupling approximation, the latter is approximated by

$$
H_{m}^{d}(Q)=[1-X(Q)] \frac{D_{s}}{D_{0}}+X(Q) H(Q),
$$

where $H(Q)$ is the hydrodynamic function of ideally monodisperse spheres of radius $R_{0}$.

To calculate $H(Q)$, we employ a self-part corrected version of the $\delta \gamma$-expansion scheme (self-part corrected $\delta \gamma$ scheme, for short). ${ }^{11}$ We will show in Sec. V that the selfpart corrected $\delta \gamma$-scheme becomes less accurate when both $\phi$ and $1 / k$ are large. This inaccuracy can be traced back to the employed self-part correction.

The hydrodynamic function can be decomposed, according to

$$
H(Q)=\frac{D_{s}}{D_{0}}+H_{d}(Q),
$$

into a self-part, $D_{s} / D_{0}$, and a distinct part, $H_{d}(Q)$, where $H_{d}(Q$ $\rightarrow \infty)=0$. Both $D_{s} / D_{0}$ and $H_{d}(Q)$ can be calculated separately using the original lowest order $\delta \gamma$-expansion results by Beenakker and Mazur. ${ }^{58-60}$ In this scheme, many-particle HIs are approximately accounted for, and only $S(Q)$ is required as static input which makes the $\delta \gamma$ method analytically tractable. A crucial point to notice here is that $S(Q)$ enters only in the $\delta \gamma$-scheme expression for $H_{d}(Q)$, whereas the corresponding $\delta \gamma$-scheme expression for $D_{s}$ is independent of the pair potential. The $\delta \gamma$-scheme provides quite accurate results for $H_{d}(Q)$ both for neutral and charged spheres, but for $D_{s}$ it is decently accurate only for neutral spheres and when $\phi \leq 0.4$. For charged spheres, a more accurate method than the $\delta \gamma$-scheme is required for calculating $D_{s}$.

In Refs. 11 and 25, it was shown that the $D_{s}$ of chargestabilized spheres can be computed to very good accuracy using the approximation of pairwise additive hydrodynamic interactions (PA-scheme), provided that $\phi \lesssim 0.15$. Like the $\delta \gamma$-scheme, the PA-scheme requires $S(Q)$, or equivalently, the radial pair-distribution function $g(r)$, as the only input. In the PA-scheme, however, three-body and higher order HIs contributions are neglected. In our PA-scheme calculations of $D_{s}$, we use numerically precise two-sphere hydrodynamic mobility tensors that include the details of near-contact lubrication, ${ }^{61-63}$ making the PA-scheme therefore exact to linear order in $\phi$. In the self-part corrected $\delta \gamma$-scheme used in this paper, $H(Q)$ is calculated according to Eq. (14), with
$D_{s} / D_{0}$ obtained from the PA-scheme, and $H_{d}(Q)$ calculated using the $\delta \gamma$-scheme. The explicit PA-scheme expression for $D_{s} / D_{0}$, and the $\delta \gamma$-scheme expression for $H_{d}(Q)$, are given, e.g., in Ref. 11.

In the special case of neutral hard spheres (HS), the thirdorder virial expansion result,

$$
\frac{D_{s}^{\mathrm{HS}}}{D_{0}} \approx 1-1.8315 \phi\left(1+0.1195 \phi-0.70 \phi^{2}\right),
$$

for $D_{s}$ has been shown to be more accurate than the corresponding $\delta \gamma$-scheme prediction. ${ }^{11}$ Equation (15) agrees very well with numerically precise accelerated Stokesian dynamics simulation ${ }^{22}$ and hydrodynamic force multipole ${ }^{64}$ results, with an accuracy better than $3 \%$ even up to the volume fraction $\phi_{f}^{\mathrm{HS}} \approx 0.49$, where the freezing transition of hard spheres occurs. For HS systems, and for high-salinity systems of charged particles alike, $H(Q)$ is favorably calculated with its $H_{d}(Q)$ part obtained from the $\delta \gamma$-scheme, and $D_{s}$ calculated using Eq. (15).

\section{RESULTS: STATIC PROPERTIES}

In the following, we distinguish between the experimental (nominal) volume fraction, $\phi^{\text {nom }}$, and the volume fraction, $\phi$, with the latter obtained as a fit parameter from our theoretical modeling of the scattering intensity. The nominal volume fraction is selected experimentally either by dilution or concentration of the deionised master suspensions with known total mass of PA colloidal particles and respective particle mass density. No distinction is made between the experimentally selected (nominal) concentration, $n_{s}^{\text {nom }}$, of added $\mathrm{KCl}$, and the salt concentration, $n_{s}$, entering the theoretical analysis via Eq. (9). The reasons why $\phi$, but not $n_{s}$, is treated as a fit parameter are discussed in detail in Subsection IV B.

In the presented study, system $\mathrm{PA}_{542}$ has been prepared with four nominal volume fractions $\phi^{\text {nom }}=0.001,0.002$, 0.004 , and 0.008 , and system $\mathrm{PA}_{1117}$ for $\phi^{\text {nom }}=0.08,0.16$, and 0.32 . For all considered volume fractions, $n_{s}^{\text {nom }}$ has been varied to examine the effect of electrostatic screening. The parameters of all samples are listed in Table I.

\section{A. Form factor}

To deduce the particle form factor of the two PA systems, we have recorded SAXS intensities for dilute samples at $\phi^{\text {nom }}<0.005$. Residual direct particle interactions were electrostatically screened by the addition of a 1-1 KCl electrolyte solution, leading to nominal salt concentrations of $n_{s}^{\text {nom }}=200$ and $500 \mu \mathrm{M}$ for systems $\mathrm{PA}_{542}$ and $\mathrm{PA}_{1117}$, respectively.

Figure 2 shows the corresponding SAXS intensities (open circles) of dilute systems $\mathrm{PA}_{542}$ and $\mathrm{PA}_{1117}$ with negligible particle correlations. The depicted intensities have distinct minima indicative of rather monodisperse particles. As shown, the intensities can be excellently fitted using Eqs. (1)-(3) with $S_{m}(Q)$ set equal to one. The best-fit form factor in Fig. 2 (solid red and blue curves) give average particle radii, $R_{0}$, of 542 and $1117 \AA$, and size polydispersities, $\Delta R / R_{0}$, of $12.1 \%$ and $5.7 \%$ for systems $\mathrm{PA}_{542}$ and $\mathrm{PA}_{1117}$, respectively. Figure 2 depicts additionally the decoupling 
TABLE I. Particle type, nominal volume fraction, $\phi^{\text {nom }}$, nominal salt concentration, $n_{s}^{\text {nom }}$, fitted volume fraction, $\phi$, fitted effective charge number, $Z$, peak wavenumber location, $Q_{m}$, and principal peak heights, $S\left(Q_{m}\right)$ and $H\left(Q_{m}\right)$, of the static structure factor and hydrodynamic function, respectively, for all investigated systems. The salt concentration, $n_{s}^{\text {nom }}$, was used as input to the MPB-RMSA fits of the experimental $S_{m}(Q)$. For the case of $n_{s}^{\text {nom }}=0$, a residual salt concentration of $n_{s}=1 \mu \mathrm{M}$ was used in the calculations. Parameters are $T=293.15 \mathrm{~K}, \epsilon=80.1$, and $R_{0}=542 \AA$ and $1117 \AA$ for system $\mathrm{PA}_{542}$ and $\mathrm{PA}_{1117}$, respectively. The arrows in the rightmost column indicate the systems where $S(Q)$ and $H(Q)$ are shown in Figs. 3, 4, 6, and 7.

\begin{tabular}{|c|c|c|c|c|c|c|c|}
\hline $\begin{array}{l}\text { Particle } \\
\text { type }\end{array}$ & $\phi^{\mathrm{nom}}$ & $\begin{array}{c}n_{\mathrm{s}}^{\mathrm{nom}} \\
{[\mathrm{mM}]}\end{array}$ & $\phi$ & $Z$ & $\begin{array}{c}Q_{m} \\
{\left[\mathrm{~nm}^{-1}\right]}\end{array}$ & $S\left(Q_{m}\right)$ & $H\left(Q_{m}\right)$ \\
\hline $\mathrm{PA}_{542}$ & 0.001 & 0 & 0.0011 & 205 & 0.009 & 1.37 & 1.04 \\
\hline $\mathrm{PA}_{542}$ & 0.001 & 0.01 & 0.0011 & 209 & 0.012 & 1.05 & 1.01 \\
\hline $\mathrm{PA}_{542}$ & 0.002 & 0 & 0.0027 & 334 & 0.011 & 2.11 & $1.11 \leftarrow$ \\
\hline $\mathrm{PA}_{542}$ & 0.002 & 0.01 & 0.0021 & 450 & 0.011 & 1.24 & $1.04 \leftarrow$ \\
\hline $\mathrm{PA}_{542}$ & 0.002 & 0.05 & 0.0021 & 750 & 0.016 & 1.05 & $1.01 \leftarrow$ \\
\hline $\mathrm{PA}_{542}$ & 0.002 & 0.1 & 0.0024 & 900 & 0.019 & 1.03 & 1.01 \\
\hline $\mathrm{PA}_{542}$ & 0.004 & 0.005 & 0.0052 & 395 & 0.014 & 1.91 & $1.12 \leftarrow$ \\
\hline $\mathrm{PA}_{542}$ & 0.004 & 0.01 & 0.0054 & 260 & 0.015 & 1.33 & $1.06 \leftarrow$ \\
\hline $\mathrm{PA}_{542}$ & 0.004 & 0.05 & 0.0030 & 500 & 0.017 & 1.06 & $1.01 \leftarrow$ \\
\hline $\mathrm{PA}_{542}$ & 0.008 & 0.005 & 0.0089 & 410 & 0.016 & 2.33 & $1.18 \leftarrow$ \\
\hline $\mathrm{PA}_{542}$ & 0.008 & 0.01 & 0.0113 & 440 & 0.018 & 2.10 & $1.17 \leftarrow$ \\
\hline $\mathrm{PA}_{542}$ & 0.008 & 0.02 & 0.0085 & 450 & 0.017 & 1.48 & 1.09 \\
\hline $\mathrm{PA}_{542}$ & 0.008 & 0.05 & 0.0055 & 680 & 0.017 & 1.15 & $1.03 \leftarrow$ \\
\hline $\mathrm{PA}_{1117}$ & 0.08 & 0 & 0.136 & 65 & 0.0213 & 1.28 & 1.03 \\
\hline $\mathrm{PA}_{1117}$ & 0.08 & 0.05 & 0.086 & 67 & 0.0236 & 1.10 & $0.93 \leftarrow$ \\
\hline $\mathrm{PA}_{1117}$ & 0.08 & 0.2 & 0.077 & 148 & 0.0236 & 1.09 & 0.97 \\
\hline $\mathrm{PA}_{1117}$ & 0.08 & 0.5 & 0.080 & 306 & 0.0236 & 1.10 & $0.97 \leftarrow$ \\
\hline $\mathrm{PA}_{1117}$ & 0.08 & 2 & 0.085 & 500 & 0.0252 & 1.09 & $0.97 \leftarrow$ \\
\hline $\mathrm{PA}_{1117}$ & 0.08 & 5 & 0.094 & 455 & 0.0263 & 1.09 & 0.97 \\
\hline $\mathrm{PA}_{1117}$ & 0.16 & 0 & 0.211 & 63 & 0.0244 & 1.32 & 0.91 \\
\hline $\mathrm{PA}_{1117}$ & 0.16 & 0.01 & 0.189 & 71 & 0.0244 & 1.30 & 0.91 \\
\hline $\mathrm{PA}_{1117}$ & 0.16 & 0.035 & 0.175 & 92 & 0.0244 & 1.29 & 0.90 \\
\hline $\mathrm{PA}_{1117}$ & 0.16 & 0.1 & 0.167 & 131 & 0.0244 & 1.29 & $0.87 \leftarrow$ \\
\hline $\mathrm{PA}_{1117}$ & 0.16 & 0.5 & 0.159 & 400 & 0.0244 & 1.29 & $0.88 \leftarrow$ \\
\hline $\mathrm{PA}_{1117}$ & 0.16 & 1 & 0.159 & 550 & 0.0248 & 1.26 & 0.88 \\
\hline $\mathrm{PA}_{1117}$ & 0.16 & 2 & 0.156 & 1100 & 0.0248 & 1.25 & $0.87 \leftarrow$ \\
\hline $\mathrm{PA}_{1117}$ & 0.16 & 3.5 & 0.145 & 1000 & 0.0255 & 1.17 & 0.83 \\
\hline $\mathrm{PA}_{1117}$ & 0.16 & 5 & 0.150 & 1000 & 0.0255 & 1.16 & 0.82 \\
\hline $\mathrm{PA}_{1117}$ & 0.32 & 0.1 & 0.360 & 260 & 0.0276 & 2.89 & $0.61 \leftarrow$ \\
\hline $\mathrm{PA}_{1117}$ & 0.32 & 0.2 & 0.364 & 285 & 0.0280 & 2.57 & 0.61 \\
\hline $\mathrm{PA}_{1117}$ & 0.32 & 0.35 & 0.351 & 292 & 0.0280 & 2.15 & $0.59 \leftarrow$ \\
\hline $\mathrm{PA}_{1117}$ & 0.32 & 3.5 & 0.325 & 500 & 0.0280 & 1.70 & 0.54 \\
\hline $\mathrm{PA}_{1117}$ & 0.32 & 5 & 0.300 & 500 & 0.0280 & 1.50 & $0.54 \leftarrow$ \\
\hline $\mathrm{PA}_{1117}$ & 0.32 & 10 & 0.281 & 500 & 0.0280 & 1.40 & 0.53 \\
\hline
\end{tabular}

factor $1-X(Q)$ of both systems, with $X(Q)$ evaluated according to Eq. (5). Good agreement is observed between $1-X(Q$ $\rightarrow 0$ ), and its low-polydispersity approximation $9\left(\Delta R / R_{0}\right)^{2}$, with the latter indicated by the horizontal green line segments at low $Q$ values.

\section{B. Structure factor}

For samples with significant particle correlations, the measurable static structure factor, $S_{m}(Q)$, was determined experimentally as the colloid concentration-weighted ratio of the SAXS- and SLS-determined mean intensity, $I_{s}(Q)$, and the corresponding low-concentration SAXS intensity data displayed in Fig. 2. Regarding the SLS measurements of $I_{s}(Q)$ at low $Q$, additional low-concentration SLS measurements were made for the static structure factor determination. A beam stop was used to block the direct beam at very low $Q$.

In Fig. 3, open circles represent the SLS-determined $S_{m}(Q)$ for nine samples of system $\mathrm{PA}_{542}$, with nominal volume fractions $\phi^{\text {nom }}=0.002,0.004$, and 0.008 , and concentrations of added $\mathrm{KCl}$ ranging from $n_{s}^{\text {nom }}=0$ (no added salt) to $0.05 \mathrm{mM}$. The panels of Fig. 3, and likewise of Figs. 4, 6 and 7 discussed further down, are ordered with respect to $\phi^{\text {nom }}$, which increases from top to bottom, and with respect to $n_{s}^{\text {nom }}$, which decreases from left to right. Hence, the strength of the inter-particle correlations increases from the top left to the bottom right panel. The SAXS $S(Q)$ for nine samples of system $\mathrm{PA}_{1117}$ are shown in Fig. 4. Here, the nominal volume fractions are $\phi^{\text {nom }}=0.08,0.16$, and 0.32 , respectively, and $n_{s}^{\text {nom }}$ is varied in between 0.05 and $5 \mathrm{mM}$.

In the MPB-RMSA fits of the experimental $S_{m}(Q)$ based on the decoupling approximation expressions in Eqs. (4) and (7), $T=293.15 K, \epsilon=80.1$, and $R_{0}=542$ or $1117 \AA$ are kept constant, while the volume fraction $\phi$ is treated as an adjustable parameter. To consider the added salt concentration, $n_{s}$, as another fit parameter would be problematic since $Z$ and $n_{s}$ opposingly influence the undulations in $S(Q)$, most prominently visible in the peak height $S\left(Q_{m}\right)$. The peak height grows with increasing $Z$, but decreases with increasing $n_{s}$. Different combinations of $Z$ and $n_{s}$ can result thus in quite similar $S(Q)$. In the theoretical analysis of the experimental $S_{m}(Q)$, it is therefore helpful to have independent control on one of the two parameters $Z$ or $n_{s}$. In the present work, $n_{s}^{\text {nom }}$ is controlled experimentally with high precision by the addition of a $\mathrm{KCl}$ solution of known concentration. Thus, in our fits of $S_{m}(Q), n_{s}$ is set equal to $n_{s}^{\text {nom }}$, leaving only the two fit parameters $\phi$ and $Z$. The best-fit values for $\phi$ and $Z$ are listed in Table I, for all systems studied. We note that $n_{s}=1 \mu \mathrm{M}$ was actually used in the case of $n_{s}^{\text {nom }}=0$, i.e., in the zero added-salt case, to account approximately for the self-dissociation of water at neutral $p \mathrm{H}\left(n_{s} \approx 0.1 \mu \mathrm{M}\right)$, and for residual electrolyte ions stemming from dissociated $\mathrm{CO}_{2}\left(n_{s}\right.$ $\lesssim 1 \mu \mathrm{M}$ ). These ions are practically inevitable in an aqueous solvent.

The red solid curves in Fig. 3 are the best-fit measurable static structure factors, $S_{m}^{d}(Q)$, obtained in decoupling approximation according to Eq. (4), in comparison with the experimental $S_{m}(Q)$ (open circles). The static structure factor, $S(Q)$, of ideally monodisperse spheres of radius $R_{0}$ entering into Eq. (4), has been computed using the analytic MPBRMSA scheme (brown dashed curves). A general observation made in Fig. 3 is that $S_{m}^{d}(Q)$ is in better agreement with the experimental data than $S(Q)$, in particular at small wavenumbers $Q<Q_{m}$, and for strongly repelling particles (large $\phi$ or low $\left.n_{s}\right)$, where $S(Q \rightarrow 0)$ has a value close to zero. According to Eq. (4), in these samples, $S_{m}^{d}(Q \rightarrow 0) \approx 1-X(Q \rightarrow 0)$. For system $\mathrm{PA}_{542}, 1-X(0) \approx 0.132$ (cf. upper green line segment in Fig. 2).

The theoretical fits of $S_{m}^{d}(Q)$ in Fig. 3 are in good overall agreement with the SLS-determined $S_{m}(Q)$, except for a systematic deviation at low $Q$. These low- $Q$ deviations can 

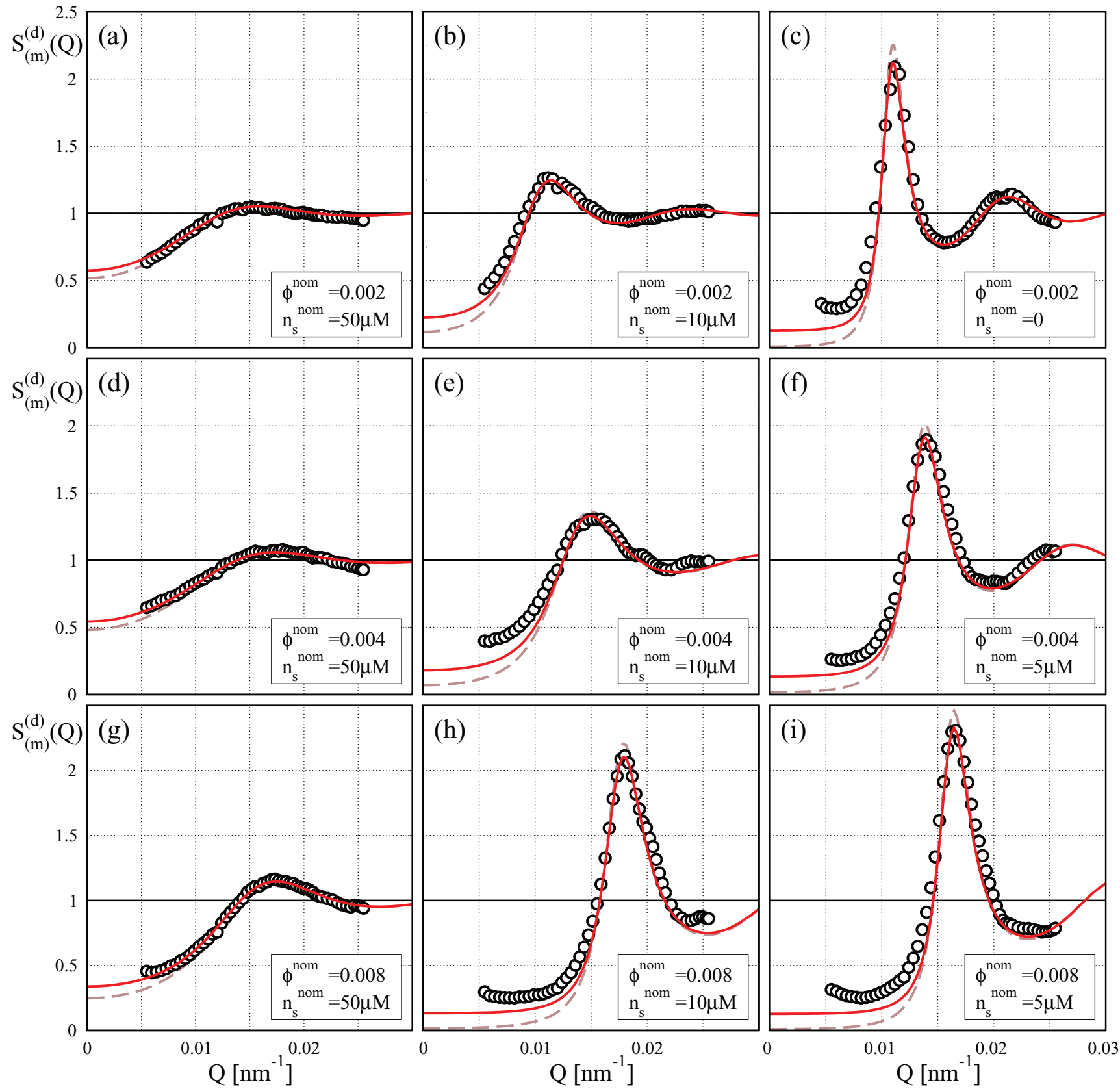

FIG. 3. Open symbols: SLS measurable static structure factors, $S_{m}(Q)$, of system $\mathrm{PA}_{542}$, at nominal volume fractions, $\phi^{\text {nom }}$, of 0.002 (top row of panels), 0.004 (center row), and 0.008 (bottom row). The nominal salt concentration, $n_{s}^{\text {nom }}$, decreases from left to right, as indicated in each panel. Dashed brown curves: ideal structure factor, $S(Q)$, in MPB-RMSA; solid red curves: measurable structure factor, $S_{m}^{d}(Q)$, in decoupling approximation. The fit parameters are listed in Table I. Identical axes scales are used in panels (a)- (i). The (measurable) hydrodynamic functions corresponding to the structure factors shown here are plotted in Fig. 6.

be partially attributed to low-concentration impurities such as dust particles. In addition, multiple scattering with more pronounced effects at small $Q$ values might add to the deviations. Moreover, there are residual solvent and mixed solvent-solute scattering contributions not accounted for in the theoretical analysis. All these left-out scattering contributions can enlarge $S_{m}(Q \approx 0)$ significantly for low-salinity systems where the ideal structure factor $S(Q \approx 0)$ is small. For a discussion of possible solvent and solvent-solute scattering corrections to the experimental structure factor, we refer to Ref. 65 .

The SAXS-determined static structure factors of the large $\mathrm{PA}_{1117}$ particles (open circles) are shown in Fig. 4. The red solid curves are best-fit MPB-RMSA results for $S(Q)$. Different from the $\mathrm{PA}_{542}$ particles in Fig. 3, where the incoherent scattering contribution at low $Q$ values is significant, the polydispersity of the big particles is so small that its influence can be disregarded. Indeed, for the samples in Fig. 4, the principal peak in $S(Q)$ occurs at $Q_{m}<0.03 \mathrm{~nm}^{-1}$. On noting (see Fig. 2) that $1-X\left(Q \leq Q_{m}\right)<0.029$ for system $\mathrm{PA}_{1117}$, this implies that for the nearly monodisperse $\mathrm{PA}_{1117}$ particles $S_{m}(Q), S_{m}^{d}(Q)$, and $S(Q)$ are practically equal. The blue dashed curves in Fig. 4 are results for $S(Q)$ for neutral hard spheres, calculated in Percus-Yevick approximation for the same $\phi$ as in the charged-particles case. For the high-salinity 

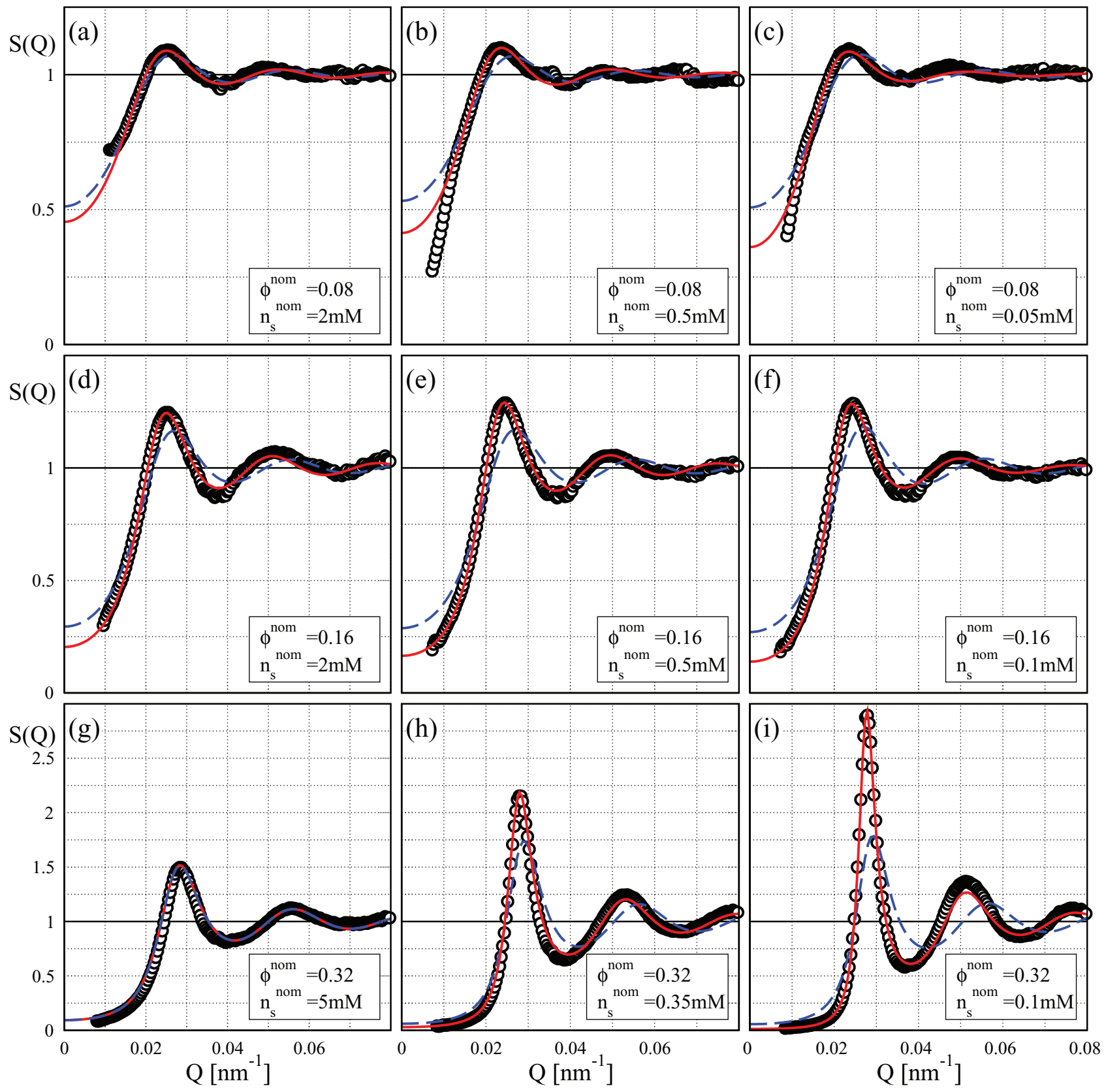

FIG. 4. Static structure factors, $S(Q)$, of system $\mathrm{PA}_{1117}$, for nominal volume fractions, $\phi^{\text {nom }}$, of 0.08 (top row of panels), 0.16 (center row), and 0.32 (bottom row). The nominal salt concentration, $n_{s}^{\text {nom }}$, decreases from left to right, as indicated. Open circles: SAXS results; red solid curves: structure factor, $S(Q)$, obtained in MPB-RMSA; blue dashed curves: Percus-Yevick result for $S(Q)$ for neutral hard spheres at the same $\phi$ as in the respective MPB-RMSA results. All fit parameters are listed in Table I. The hydrodynamic functions corresponding to the $S(Q)$ 's are plotted in Fig. 7. The horizontal axis scale is the same in each panel. The vertical axis scale is the same in each row of panels. Unlike the $\mathrm{PA}_{542}$ samples structure factors plotted in Fig. $3, S(Q), S_{m}(Q)$, and $S_{m}^{d}(Q)$ are practically indistinguishable for the low-polydispersity $\mathrm{PA}_{1117}$ system.

sample (g), the charged-sphere and neutral hard-sphere structure factors are practically equal, indicating that the electrostatic repulsion in this system is fully screened. The same conclusion applies to the sample at $\phi^{\text {nom }}=0.32$, which has an even higher salt concentration of $10 \mathrm{mM}$ (its $S(Q)$ is not shown in Fig. 4, but its parameters are listed in Table I).

We note from Table I that for both particulate systems $\mathrm{PA}_{542}$ and $\mathrm{PA}_{1117}$, the deduced effective charge number, $Z$, is overall increasing with increasing volume fraction, and increasing concentration of added electrolyte. This is consistent with cell-model based charge renormalization calcula- tions predicting the same trends for not too small volume fractions. ${ }^{43,66}$ The smaller effective charge values for the $\mathrm{PA}_{1117}$ samples at low salinities could be due to the additional growth step performed in the synthesis process of this particles, resulting possibly in a smaller number of charged sulfate groups per unit of surface area.

According to Table I, the best-fit volume fractions $\phi$ are overall in good agreement with the nominal values $\phi^{\text {nom }}$. For the $\mathrm{PA}_{1117}$ samples with larger $\phi^{\text {nom }}$, a slight decrease of $\phi$ with increasing $n_{s}$ can be observed. The reason for this is unclear, in particular, since there is no indication for the 
formation of aggregates even at the largest considered salt concentration.

\section{Limiting scaling relations for the nearest neighbor shell}

According to Table I, the principal peak wavenumber location, $Q_{m}$, of the static structure factor shifts to larger values with increasing $\phi^{\text {nom }}$, indicative of a compression of the nearest neighbor shell radius, $R_{m} \sim 2 \pi / Q_{m}$, with increasing particle concentration. A precise definition of the nearest neighbor shell radius is given by the principal peak position, $r_{m}$, of the radial distribution function $g(r)$. For samples of equal $\phi^{\text {nom }}, Q_{m}$ shifts to larger values with increasing electrolyte concentration, as expected. Only for the sample (h) in Fig. 3, with $\phi^{\text {nom }}=0.008$ and $n_{s}^{\text {nom }}=10 \mu \mathrm{M}$, is this trend violated. This can be attributed to experimental variations in the particle concentration during sample preparation, reflected in the relatively large differences between $\phi$ and $\phi^{\text {nom }}$ in row 11 of Table I.

In Fig. 5, $Q_{m} \times\left(2 R_{0}\right)$ and $r_{m} /\left(2 R_{0}\right)$ are displayed as functions of $\phi$, for all explored systems. Filled symbols are the findings for the seven systems of lowest salt concentration, $n_{s}^{\text {nom }}$, at a given $\phi^{\text {nom }}$. These are the samples whose parameters are given in rows $1,3,7,10,14,20$, and 29 of Table I. As expected, the nearest neighbor shell radius, $r_{m}$, decreases with increasing $\phi$. For non-overlapping particles, $r_{m} /\left(2 R_{0}\right)$ $\geq 1$, with the limiting ratio $r_{m} /\left(2 R_{0}\right)=1$ valid for neutral hard spheres. The hard-sphere limit is almost reached for the $\mathrm{PA}_{1117}$ samples with the largest values of $n_{s}^{\text {nom }}$ (open red squares in Fig. 5). While $S(Q)$ and $g(r)$ are Fourier transform pairs, there is in general no simple one-to-one relation between $r_{m}$ and $Q_{m}$. However, $Q_{m} \times\left(2 R_{0}\right) \approx 2 \pi$ for the $\mathrm{PA}_{1117}$

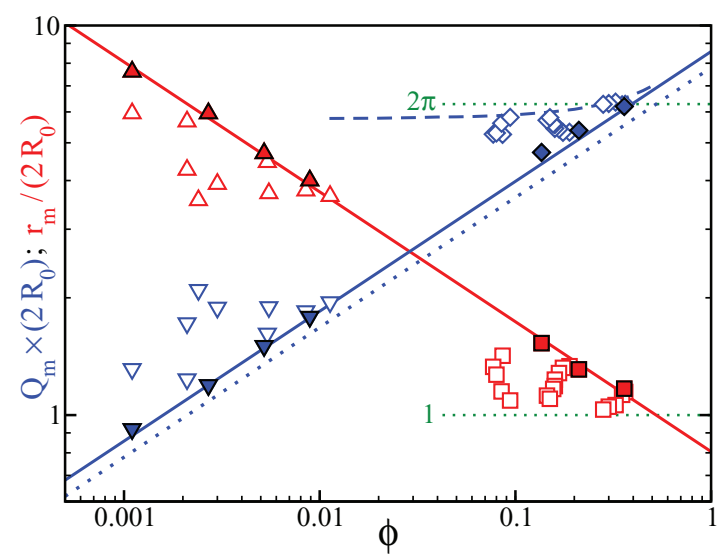

FIG. 5. Red squares and red upward triangles: principal peak position, $r_{\mathrm{m}}$, of the MPB-RMSA radial distribution function $g(r)$. Blue diamonds and blue downward triangles: principal peak position, $Q_{m}$, of $S(Q)$. Both $r_{m}$ and $Q_{m}$ are normalized with the mean particle diameter, $2 R_{0}$, and plotted on a double-logarithmic scale as functions of the fit volume fraction $\phi$. Triangles: $\mathrm{PA}_{542}$ samples. Squares and diamonds: $\mathrm{PA}_{1117}$ samples. Solid red and blue lines: limiting $\phi$-scaling expressions in Eqs. (16) and (17) for $r_{m}^{\max } /\left(2 R_{0}\right)$ and $Q_{m}^{\min } \times\left(2 R_{0}\right)$. Dotted blue line: Eq. (17), with the factor 2.2 replaced by 2.0. Dashed blue curve: $Q_{m}^{\mathrm{HS}} \times\left(2 R_{0}\right)$, where $Q_{m}^{\mathrm{HS}}$ is the peak position of the Percus-Yevick neutral hard-sphere $S(Q)$. The data points comprise all systems in Table I. For each nominal volume fraction, $\phi^{\text {nom }}$, the system of lowest salt concentration, $n_{s}^{\text {nom }}$, is represented by a filled symbol. samples with largest considered values of $n_{s}^{\text {nom }}$ (open blue diamonds in Fig. 5). Note here that the product $Q_{m} \times\left(2 R_{0}\right)$ slightly exceeds $2 \pi$ for $\phi \gtrsim 0.3$, and for high salt concentrations (rightmost open diamonds). A strict upper limit for $Q_{m}$, at given $\phi$, is provided by the wavenumber, $Q_{m}^{\mathrm{HS}}$, where the $S(Q)$ of neutral hard spheres attains its principal maximum. In Fig. 5, $Q_{m}^{\mathrm{HS}}$ predicted in Percus-Yevick approximation is represented by the dashed blue curve.

As noticed from Fig. 5, an upper limit for $r_{m}$ is provided by the simple-cubic mean geometrical distance $r_{m}^{\max }=n^{-1 / 3}$. For strongly repelling (low-salinity) systems of charged particles interacting by the HSY pair potential in Eq. (7), it has been shown in Ref. 9 that $r_{m} \approx r_{m}^{\max }$ and $Q_{m} \approx Q_{m}^{\min }$ $=2.2 \pi n^{1 / 3}$, where

$$
r_{m}^{\max } /\left(2 R_{0}\right)=(\pi / 6)^{1 / 3} \phi^{-1 / 3}
$$

and

$$
Q_{m}^{\min } \times\left(2 R_{0}\right)=2.2 \times 6^{1 / 3} \times \pi^{2 / 3} \times \phi^{1 / 3} .
$$

The $\phi$-scaling expressions in Eqs. (16) and (17) are represented in Fig. 5 by the solid red and blue lines. Due to the factor 2.2 appearing in Eq. (17), $Q_{m}^{\min }$ is not equal to $2.0 \pi / r_{m}^{\max }$. Instead, the relation $Q_{m}^{\min }=2.2 \pi / r_{m}^{\max }$ applies. Our lowestsalt concentration samples which cover three orders of magnitude in $\phi$ (filled symbols), have peak positions in excellent agreement with Eqs. (16) and (17) for the value 2.2 (blue solid line), but not for the value 2.0 (blue dotted line).

Note that our experimental-theoretical findings for the concentration dependence of $r_{m}$ and $Q_{m}$ are in qualitative agreement with related studies for dilute suspensions of charged silica spheres, ${ }^{67,68}$ where, however, the erroneous prefactor 2.0 has been used in Eq. (17).

At fixed electrolyte concentration, $S\left(Q_{m}\right)$ increases with increasing $\phi$ (see Table I and Figs. 3 and 4), due to the enlarged particle correlations. Different from that, for fixed volume fraction, an increase in $n_{s}^{\text {nom }}$ results in a decrease of $S\left(Q_{m}\right)$, due to the stronger screening of the electrostatic interactions. The decrease in $S\left(Q_{m}\right)$ is accompanied by a principal peak broadening, i.e., by a more diffuse nearest neighbor cage.

\section{RESULTS: DYNAMIC PROPERTIES}

We compare our experimental results for the short-time diffusion properties with predictions by the self-part corrected $\delta \gamma$-scheme, which requires $S(Q)$ as the only input. The latter is given by the MPB-RMSA fits of the SLS and SAXS data discussed earlier. Thus, our theoretical results for $D(Q)$, $H(Q), D_{s}, D_{c g e}$, and $D_{c}$ presented in the following are free of adjustable parameters. Therefore, deviations between our experimental data and theoretical predictions for the short-time diffusion properties are indicative of inaccuracies caused by the approximate treatment of many-body HIs and the invoked decoupling approximation.

All short-time diffusion data presented are rendered dimensionless by dividing them by the translational free diffusion coefficient $D_{0}$, deduced from the short-time decay of $g_{1}(Q, \tau)$ at high dilution where $D_{0}=D_{s} \equiv D(Q)$ and $S(Q) \equiv 1$ within experimental scatter, so that $g_{1}(Q, \tau)$ 

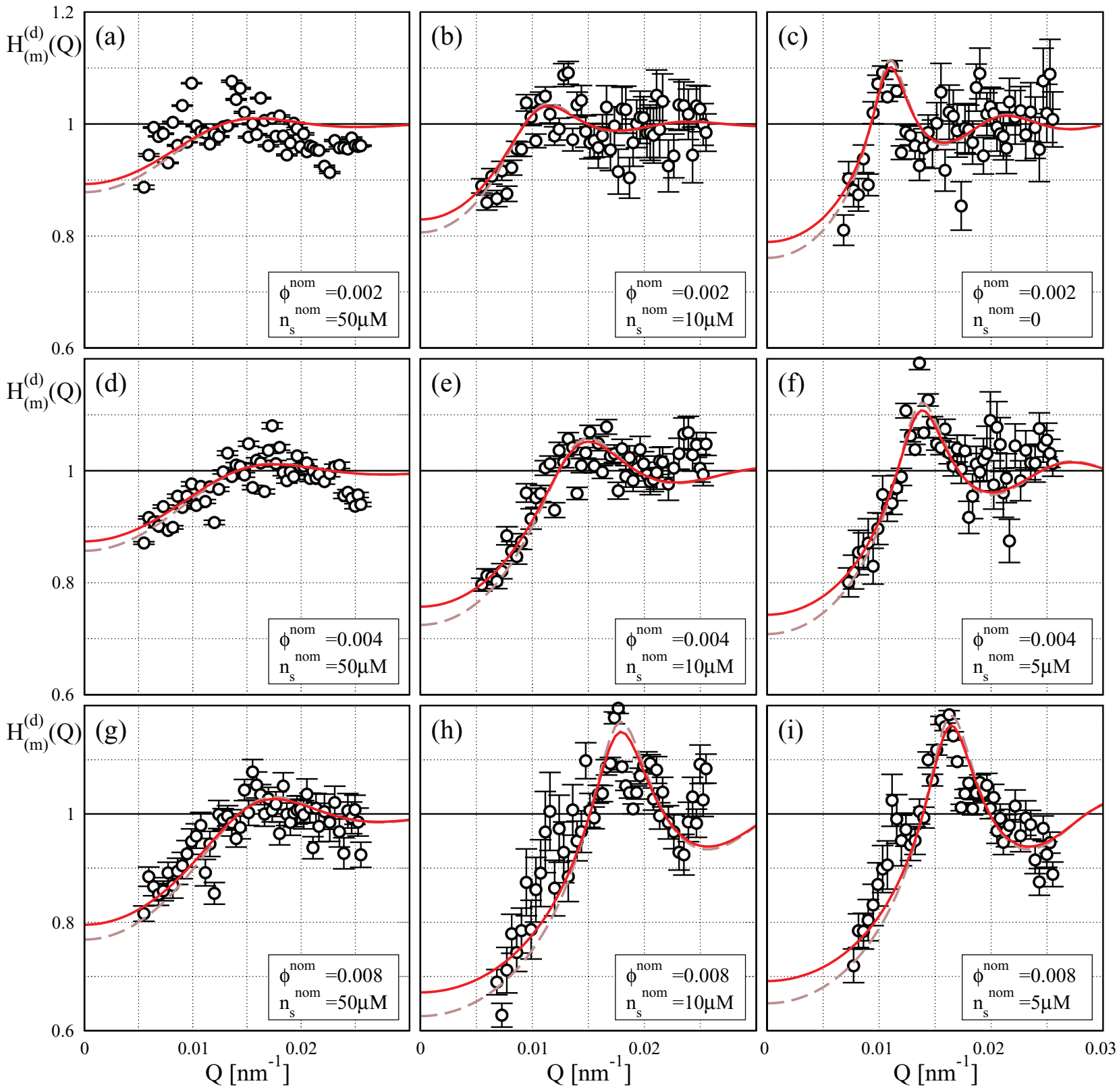

FIG. 6. Open circles: DLS/SLS-determined measurable hydrodynamic function, $H_{m}(Q)$, of the $\mathrm{PA}_{542}$ samples with nominal volume fraction, $\phi^{\text {nom }}$,

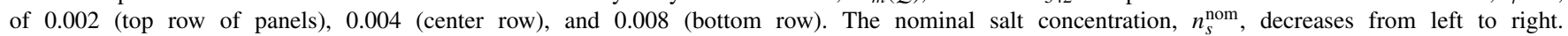
Dashed brown curves: hydrodynamic function, $H(Q)$, of ideally monodisperse particles calculated using the self-part corrected $\delta \gamma$-scheme; solid red curves: measurable hydrodynamic function, $H_{m}^{d}(Q)$, calculated in decoupling approximation according to Eq. (13). System parameters are listed in Table I. The corresponding (measurable) structure factors, $S_{m}(Q), S_{m}^{d}(Q)$, and $S(Q)$, have been plotted in Fig. 3. Identical axes scales are used in all panels.

$\propto \exp \left\{-Q^{2} D_{0} \tau\right\}$. We have obtained in this way the experimental mean values $D_{0}=3.49 \times 10^{-12} \mathrm{~m}^{2} / \mathrm{s}$ and 1.90 $\times 10^{-12} \mathrm{~m}^{2} / \mathrm{s}$ for the $\mathrm{PA}_{542}$ and $\mathrm{PA}_{1117}$ particles, respectively, at $T=20^{\circ} \mathrm{C}$.

\section{A. Hydrodynamic function}

The (measurable) hydrodynamic functions of the $\mathrm{PA}_{542}$ and $\mathrm{PA}_{1117}$ samples are displayed in Figs. 6 and 7, respectively. Open circles represent experimental data for $H_{m}(Q)$, obtained by multiplying the DLS and XPCS determined measurable short-time diffusion function, $D_{m}(Q)$, with the SLS and SAXS determined measurable static structure factors depicted in Figs. 3 and 4, and by dividing through the experimental values for $D_{0}$. For the $\mathrm{PA}_{542}$ system samples, where incoherent scattering caused by the larger polydispersity is significant, $D_{m}(Q)$ has been extracted experimentally by a first cumulant analysis according to Eq. (12).

The experimental data for $H_{m}(Q)$ in Fig. 6 are compared with our theoretical results in decoupling approximation, related to the hydrodynamic function $H(Q)$ of ideally monodisperse spheres of radius $R_{0}$ by Eq. (13). We recall from Sec. IV that the incoherent scattering contribution is negligible for the nearly monodisperse $\mathrm{PA}_{1117}$ particles where $X(Q)$ 

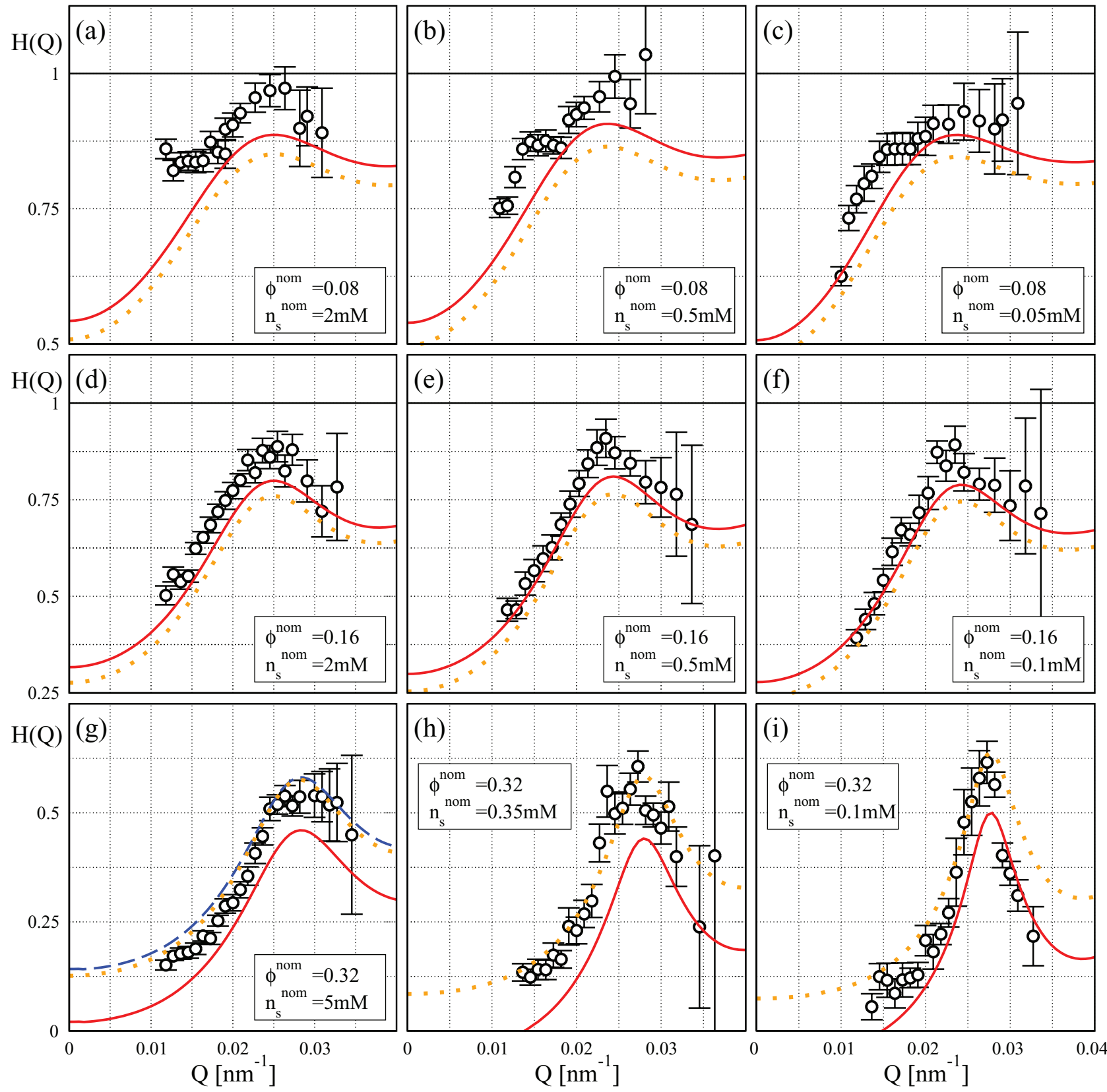

FIG. 7. Hydrodynamic function, $H(Q)$, of $\mathrm{PA}_{1117}$ samples with nominal volume fraction, $\phi^{\text {nom }}=0.08$ (top row of panels), 0.16 (center row), and 0.32 (bottom row). The nominal salt concentration, $n_{s}^{\text {nom }}$, decreases from left to right. Open circles: XPCS and SAXS data; dotted orange curves: $H(Q)$ according to the uncorrected $\delta \gamma$-scheme. Solid red curves: $H(Q)$ obtained from the self-part corrected $\delta \gamma$-scheme, with $D_{s} / D_{0}$ calculated using the PA-scheme. Dashed blue curve: $H(Q)$ in the self-part corrected $\delta \gamma$-scheme, but with $D_{s} / D_{0}$ taken as that of neutral hard spheres according to Eq. (15). The system parameters are listed in Table I, and the corresponding structure factors are shown in Fig. 4. The horizontal axis scale is identical in each panel, and the vertical $H(Q)$ axis scale is identical in each row of panels. The displayed $Q$-range is half of that in Fig. 4. Unlike the $\mathrm{PA}_{542}$ samples hydrodynamic functions plotted in Fig. 6, $H(Q)$, $H_{m}(Q)$, and $H_{m}^{d}(Q)$ are practically indistinguishable for the low-polydispersity $\mathrm{PA}_{1117}$ system.

$\approx 1$ in the full $Q$-interval of Fig. 7. For the $\mathrm{PA}_{1117}$ system, we have therefore determined $D(Q)$ experimentally from fitting the short-time decay of the normalized field autocorrelation function using $g_{1}(Q, \tau) \propto \exp \left\{-Q^{2} D(Q) \tau\right\}$. To avoid radiation damage of the $\mathrm{PA}_{1117}$ samples, XPCS measurements were performed in laminar flow, with scattering wavevector $\mathbf{Q}$ selected perpendicular to the flow direction. The flow measurements are affected by decorrelation arising from the transit time of the particles across the scattering volume. This has been accounted for by the additional decorrelation factor $g_{1}(Q, \tau) \propto \exp \left[-\left(v_{t r} \tau\right)^{2}\right]$ where the transit rate, $v_{t r}$, depends on the (mean) flow speed and the illuminated sample area. For details see Ref. 69.

The red solid lines in Fig. 6 are theoretical results for $H_{m}^{d}(Q)$ obtained in decoupling approximation according to Eq. (13). The invoked $H(Q)$ of ideally monodisperse particles is represented by the brown dashed curves, obtained using the self-part corrected $\delta \gamma$-scheme with $D_{s} / D_{0}$ calculated from the PA-scheme. For the structure factor input, the MPB-RMSA $S(Q)$ 's depicted in Fig. 3 have been used.

The red solid curves in Fig. 7 are the results for $H(Q)$ by the self-part corrected $\delta \gamma$-scheme in conjunction with the 
PA-scheme results for $D_{s} / D_{0}$. The corresponding MPBRMSA $S(Q)$, used as static input, are shown in Fig. 4. The orange dotted curves in Fig. 7 are results for $H(Q)$ obtained using Beenakker and Mazur's original $\delta \gamma$-scheme. The blue dashed curve in panel (g) of Fig. 7 is the $H(Q)$ from the selfpart corrected $\delta \gamma$-scheme, however with $D_{s} / D_{0}$ obtained from the neutral hard-sphere expression in Eq. (15).

For the samples with $\phi^{\text {nom }} \leq 0.16$ (Fig. 6 and panels (a)(f) in Fig. 7), there is good agreement between the experimental $H_{m}(Q)$ and $H(Q)$ and the theoretical predictions by the self-part corrected $\delta \gamma$-scheme, with PA-scheme $D_{s} / D_{0}$. Significant deviations are seen, however, for the most concentrated samples in Fig. 7 where $\phi^{\text {nom }}=0.32$. The reason for these deviations is the known failure of the PA-scheme to correctly predict $D_{s} / D_{0}$ for volume fractions $\phi \gtrsim 0.15$, when three-body and higher order HIs contribute significantly to the short-time self-diffusion coefficient. ${ }^{11}$ Higher order HIs influences on $D_{s}$, left out in the PA-scheme, give rise to the shielding of the HIs between a pair of particles by interlaced third particles or particle clusters. Hydrodynamic shielding reduces the strength, but not the range of the HIs, and it gives rise to an increase of $D_{s} / D_{0}$ relative to the PA-scheme predicted values. This explains why the experimental $H(Q)$ of the $\mathrm{PA}_{1117}$ samples at $\phi^{\text {nom }}=0.32$ are overall underestimated by the selfpart corrected $\delta \gamma$-scheme prediction with PA-scheme $D_{s} / D_{0}$. The underestimation of $D_{s} / D_{0}$ by the PA-scheme at the considered large $\phi$ values is so strong that unphysical negative values of $H(Q)$ at low $Q$ are predicted for the samples in panels (h) and (i) of Fig. 7. Note, however, from Eq. (14) and panels (g)-(i) in Fig. 7, that the distinct part, $H_{d}(Q)$, of $H(Q)$ is still predicted with good accuracy by the $\delta \gamma$-scheme even at $\phi^{\text {nom }}=0.32$.

These observations suggest that the self-part corrected $\delta \gamma$-scheme, with PA-scheme $D_{s} / D_{0}$ input, is reliably applicable for $\phi \lesssim 0.15$ only. Indeed, at $\phi^{\text {nom }}=0.32$ the uncorrected $\delta \gamma$-scheme predictions for $H(Q)$ (orange dotted curves in Fig. 7) are somewhat fortuitously in better agreement with the experimental data. On first sight, one may conclude thus that the uncorrected $\delta \gamma$-scheme should be used in place of the corrected $\delta \gamma$-scheme whenever the $\delta \gamma$-scheme prediction for $D_{s} / D_{0}$ is larger than the one by the corresponding PAscheme. Stated alternatively, for a given potential parameter set $(\gamma, k, \phi)$, one could calculate $D_{s} / D_{0}$ both using the $\delta \gamma$ - and the PA-schemes, adding the larger of the two predictions for $D_{s} / D_{0}$ to the $\delta \gamma$-scheme result for $H_{d}(Q)$. While such an $a d$ hoc approach has some empirical justification, from a theoretical point of view it is unsatisfying for the following reason: Switching from the PA- to the $\delta \gamma$-scheme result for $D_{s} / D_{0}$ at some volume fraction $\phi^{*}$, where the predicted values cross over, induces an unphysical kink (at $\phi^{*}$ ) in $D_{s}(\phi)$. Moreover, it is unsatisfying that the invoked zeroth-order $\delta \gamma$-scheme expression for $D_{s} / D_{0}$ has no dependence on the form of the pair potential.

Thus, in place of an ad hoc switching between the PA and $\delta \gamma$-scheme results for $D_{s} / D_{0}$, a theoretical input for $D_{s} / D_{0}$ reliable at all fluid-state volume fractions is on request. Without resorting to numerically expensive simulations, such an input is available to date only for the special case of neutral hard spheres, for which Eq. (15) can be applied to very good accuracy for concentrations up to the hard-sphere freezing transition value. For the $\mathrm{PA}_{1117}$ sample at $\phi^{\text {nom }}=0.32$ and $n_{s}^{\text {nom }} \geq 5 \mathrm{mM}$ (the two lowermost entries of Table I), Eq. (15) for $D_{s} / D_{0}$ is a viable input indeed, on recalling from our discussion of system (g) in Fig. 4 that, due to the high salt content, the pair correlations in these systems are practically indistinguishable from those of neutral hard spheres. In panel (g) of Fig. 7 the blue dashed curve is $H(Q)$ with $H_{d}(Q)$ calculated in the $\delta \gamma$-scheme and $D_{s} / D_{0}$ according to Eq. (15). It is in excellent agreement with the experimental hydrodynamic function.

No simple analytic method is available to date allowing for an accurate calculation of the $D_{s}$ for low-salinity systems with $\phi \gtrsim 0.15$ showing long-ranged electrostatic repulsion. For the $\mathrm{PA}_{1117}$ samples at $\phi^{\mathrm{nom}}=0.32$ and lower salt concentrations, what can be currently done is to invoke elaborate computer simulations ${ }^{22}$ for $D_{s} / D_{0}$, to use the non-modified $\delta \gamma$-scheme for $H(Q)$, or to treat $D_{s} / D_{0}$ simply as an adjustable parameter.

\section{B. Wavenumber dependent diffusion and scaling relations}

We proceed by discussing the concentration dependence of the normalized collective diffusion coefficient $D_{c} / D_{0}$ $=H(Q \rightarrow 0)$, the normalized short-time self-diffusion coefficient $D_{s} / D_{0}=H(Q \rightarrow \infty)$, and the hydrodynamic function peak value $H\left(Q \approx Q_{m}\right)$. See here panels (a), (b), and (c) in Fig. 8 for the experimental values. In each panel, the $\mathrm{PA}_{542}$ sample data are represented by triangles, and $\mathrm{PA}_{1117}$ data by squares. As in Fig. 5, filled symbols in Fig. 8 are results for the seven systems where $n_{s}^{\text {nom }}$ is lowest at a given $\phi^{\text {nom }}$, with parameters listed in rows $1,3,7,10,14,20$, and 29 of Table I.

The collective diffusion coefficient $D_{c}$, which quantifies the (initial) decay rate of thermally induced density fluctuations of macroscopic wavelengths, is difficult to access experimentally since it invokes a delicate zero$Q$ extrapolation of $D(Q)$. Incoherent scattering contributions at low $Q$ cause significant deviations between the experimental values, and the theoretical predictions for $D(Q)$ and $S(Q)$ without polydispersity. The most pronounced low- $Q$ deviations occur at low salinity between the ideal structure factor $S(Q)$ and the measurable static structure factor (see Fig. 3). The sample with the strongest deviations between the two functions is the $\mathrm{PA}_{542}$ sample at $\phi^{\text {nom }}=0.002$ and $n_{s}^{\text {nom }}=0$ (panel (c)) in Figs. 3 and 6. For this system, the theoretical prediction is $D_{c} / D_{0}=94$, as indicated by the uppermost filled triangle in panel (a) of Fig. 8. If one would try to determine $D_{c} / D_{0}$ using the ratio of the experimental values of $H_{m}(Q \approx 0)=0.81$ and $S_{m}(Q \approx 0)=0.29$, the erroneous result $D_{c} / D_{0}=2.80$ would be obtained, differing from the theoretical value 94 by a large factor of 34 . Hence, a theoretical analysis of the experimental data is indispensable for a reliable determination of the (mean) collective diffusion coefficient. Note that the short-time collective diffusion coefficient discussed here is only slightly larger than its longtime counterpart. ${ }^{22}$ Different from that, the long-time selfdiffusion coefficient in concentrated systems is substantially 


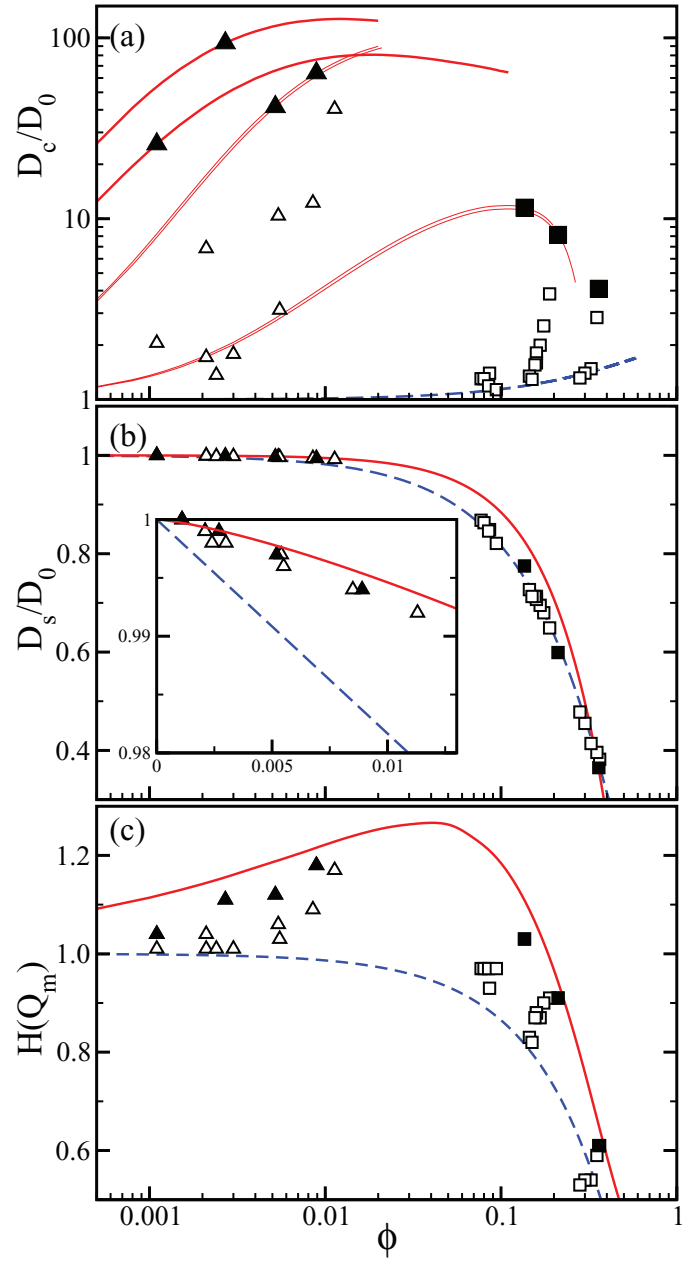

FIG. 8. (a) Normalized (short-time) mean collective diffusion coefficient $D_{c} / D_{0}$. (b) Normalized short-time translational self-diffusion coefficient $D_{s} / D_{0}$. (c) Hydrodynamic function peak value $H\left(Q_{m}\right)$. All quantities are plotted as functions of the fit volume fraction $\phi$. Triangles: PA $_{542}$ samples; Squares: $\mathrm{PA}_{1117}$ samples. For each $\phi^{\text {nom }}$, the sample of lowest nominal salt concentration is represented by a filled symbol. Dashed blue curves in (a)(c) are the neutral hard-sphere results in Eqs. (18), (15) and (20), respectively. Red solid curves are our theoretical results for highly charged particles at low salinity. The solid curves in (a) were obtained using the self-part corrected $\delta \gamma$-scheme with PA-scheme $D_{s} / D_{0}$ for fixed values of $Z$ and $n_{s}$, and for the six samples with parameters listed in rows $1,3,7,10,14$, and 20 of Table I. In (b), the solid curve represents the scaling expression for the $D_{s} / D_{0}$ of charged particles in Eq. (19), using $a_{t}=2.5$. The solid curve in (c) is the limiting freezing line result for the $H\left(Q_{m}\right)$ of strongly repelling charged particles, calculated according to Ref. 24.

smaller than $D_{s}$, down to a value of $0.1 D_{s}$ at the freezing transition point. ${ }^{70}$

The coefficient $D_{c} / D_{0}$ of charged colloidal spheres increases with decreasing salt concentration. For very high salt concentrations (lowermost open squares in panel (a) of Fig. 8), the experimental $D_{c} / D_{0}$ are close to the truncated second-order virial expansion result,

$$
\frac{D_{c}^{\mathrm{HS}}}{D_{0}} \approx 1+1.454 \phi-0.45 \phi^{2},
$$

for neutral hard spheres, which is known from comparison with simulation ${ }^{22}$ to be decently accurate even for large volume fractions up to the freezing transition value $\phi_{f}^{H S} \approx 0.49$.
Equation (18) is represented by the dashed blue curve in panel (a) of Fig. 8.

A similarly simple expression for the upper limiting boundary for the $D_{c} / D_{0}$ of charged particles interacting by the HSY pair potential is not known. For lower salinity systems, $D_{c}(\phi) / D_{0}$ can pass through a maximum as a function of $\phi$ (for constant $Z>0$ ), ${ }^{12-16}$ increasing initially steeply from the infinite dilution value of one. The strong low- $\phi$ increase of $D_{c}(\phi)$ is triggered by the strong decrease of the reduced isothermal osmotic compressibility, $S(Q \rightarrow 0)$, with increasing $\phi$. With further increasing $\phi$, HIs can slow collective diffusion to such an extent that $D_{c}(\phi)$ passes through a maximum at some $\phi^{\mathrm{m}}$. With increasing $Z$ or decreasing $n_{s}$, the maximum value $D_{c}\left(\phi^{\mathrm{m}}\right) / D_{0}$ increases, whereas $\phi^{\mathrm{m}}\left(Z, n_{s}\right)$ decreases.

The six solid red curves in panel (a) of Fig. 8 are the self-part corrected $\delta \gamma$-scheme predictions for $D_{c} / D_{0}$, with PAscheme input for $D_{s} / D_{0}$. The values for $Z$ and $n_{s}$ are taken from rows $1,3,7,10,14$, and 20 of Table I and are kept constant for varying $\phi$. The lowermost two solid red curves in panel (a) are nearly coincident owing to the very similar values of $Z$ and $n_{s}$ in rows 14 and 20 of the table. For the same reason, the third and fourth solid red curve as counted from the bottom of panel (a) are nearly coincident. No theoretical curve for $D_{c} / D_{0}$ passes through the rightmost filled square in panel (a) of Fig. 8, corresponding to a sample of volume fraction $\phi=0.36$, for the earlier discussed reason that the PA-scheme input for $D_{s} / D_{0}$ is not applicable at such a large volume fraction.

We discuss now the results for $D_{s}$ in Fig. 8 (see panel (b)). The triangles and squares are the PA-scheme results for $D_{s} / D_{0}$, except for the $\mathrm{PA}_{1117}$ sample at $\phi^{\text {nom }}=0.32$ (rightmost squares). Since the PA-scheme result for $D_{s} / D_{0}$ are not reliable at large $\phi$, for samples with $\phi^{\text {nom }}=0.32$ we have treated $D_{s} / D_{0}$ simply as a fit parameter determined by matching $H_{d}^{\delta \gamma}(Q)+D_{s} / D_{0}$ to the experimental $H(Q)$, three of which are depicted in panels (g)-(i) of Fig. 7.

The lower limiting boundary for the $D_{s} / D_{0}$ of charged particles with HSY pair interaction is provided by the neutral hard-sphere virial result in Eq. (15) (dashed blue curve in panel (b)) of Fig. 8). Electrostatic repulsion disfavors nearcontact particle configurations, thereby suppressing near-field HIs and causing $D_{s} / D_{0}$ to grow with increasing interaction parameter $\gamma$ and decreasing screening parameter $k$. The values for $D_{s} / D_{0}$ of strongly repelling HSY-type particles are limited from above by the $\phi$-scaling form

$$
\frac{D_{s}^{\max }}{D_{0}}=1-a_{t} \phi^{4 / 3}
$$

of fractional exponent $4 / 3$, and with coefficient $a_{t} \approx 2.5-2.9$, depending to some extend on particle charge and size. ${ }^{14,25,71}$ Equation (19) has been derived on basis of leading-order farfield HIs contributions only, giving therefore a lower estimate for the hydrodynamic slowing of self-diffusion. The exponent $4 / 3$ is a consequence of the $r_{m}^{\max } \propto \phi^{-1 / 3}$ scaling of the nearest neighbor cage radius. The solid red line in panel (b) of Fig. 8 represents Eq. (19) for $a_{t}=2.5$.

We finally discuss the concentration dependence of $H\left(Q_{m}\right)$ depicted in panel (c) of Fig. 8. Like $S(Q)$, the hydrodynamic function has its maximum at $Q \approx Q_{m}$. The peak 
value $H\left(Q_{m}\right)$ quantifies the influence of HIs on the kinetic part of short-time concentration fluctuations with wavelength comparable to the nearest neighbor cage radius. The cagediffusion coefficient, $D_{\text {cge }}=D\left(Q_{m}\right)$, is lowered by HIs when $H\left(Q_{m}\right)<1$, and enhanced when $H\left(Q_{m}\right)>1$. Like $S\left(Q_{m}\right)$, $H\left(Q_{m}\right)$ is largest for the lowest electrolyte concentration. From Fig. 7, from panel (c) of Fig. 8, and from Table I we note that $H\left(Q_{m}\right)<1$ for nearly all $\mathrm{PA}_{1117}$ samples where $\phi^{\text {nom }} \geq 0.08$. The only exception is made by the sample with $\phi^{\text {nom }}=0.08$ and $n_{s}^{\text {nom }}=0$, where $H\left(Q_{m}\right)=1.03$ (not shown in Fig. 7). In contrast, values $H\left(Q_{m}\right)>1$ are observed for all $\mathrm{PA}_{542}$ samples with $\phi^{\text {nom }} \leq 0.008$.

Our experimental observation of the hydrodynamic enhancement of $H\left(Q_{m}\right)$ at low salinity is in accord with our theoretical predictions (solid red lines in Fig. 6). It is also in line with experimental, simulation, and theory results for the $H(Q)$ of charged colloidal particles reported elsewhere. ${ }^{11,17-25}$ In particular, no unusually small, so-called ultra slow values of $H\left(Q_{m}\right)$, as reported in Ref. 72 in conflict with the theoretical predictions, have been found in our study.

A lower limiting curve for $H\left(Q_{m}\right)$ is provided by the firstorder virial result,

$$
H^{\mathrm{HS}}\left(Q_{m}\right)=1-1.35 \phi,
$$

for neutral hard spheres. It has been shown ${ }^{11,22,73}$ that this linear form describes the peak height excellently even up to the hard-sphere freezing transition concentration. Equation (20) is depicted by the dashed blue line in panel (c) of Fig. 8.

An upper limiting curve for the $H\left(Q_{m}\right)$ of HSY-type particles has been obtained in Ref. 24. It is represented by the red solid curve in panel (c) of Fig. 8. This upper limiting curve has been constructed using the $\delta \gamma$-scheme with RMSA static structure factor input, by sampling over the full set of pair potential parameters $(\gamma, k$, and $\phi)$ in the liquid-phase regime, and using the empirical Hansen-Verlet criterion value, $S\left(Q_{m}\right)$ $\approx 3.1$, for the onset of freezing of strongly repelling charged particles. ${ }^{74-77}$ The limiting curve is not appreciably changed when in place of the RMSA $S(Q)$, the more accurate MPBRMSA or Rogers-Young $S(Q)$ 's are used as static input.

Nearly all our experimentally determined hydrodynamic function peak values are located between the discussed upper and lower limiting curves. Only for the two samples with $\phi^{\text {nom }}=0.32$, and $n_{s}=5$ and $10 \mathrm{mM}$, does $H\left(Q_{m}\right)$ slightly undershoot the hard-sphere limiting curve. For these two samples, $\phi$ obtained by the model fit to $S(Q)$ is also slightly smaller than the experimental value $\phi^{\text {nom }}=0.32$. Thus, the observed slight undershooting is likely due to some inaccuracy in the actual volume fraction.

\section{CONCLUSIONS}

We have presented a comprehensive experimentaltheoretical study of the equilibrium microstructure and wavenumber-resolved short-time diffusion properties in aqueous suspensions of charged PA spheres, representative of charge-stabilized colloidal suspensions. In the synthesis and preparation of our samples, the parameters $R_{0}, \Delta R / R_{0}, \phi^{\mathrm{nom}}$, and $n_{s}^{\text {nom }}$ have been varied over broad ranges, so that essentially the complete fluid phase regime has been explored.
For the less concentrated samples, we have collected scattering data using experimentally less demanding static and dynamic light scattering experiments. For the more concentrated, opaque-looking samples, where light scattering experiments are infeasible due to multiple scattering, we have recorded SAXS and XPCS data for colloid volume fractions up to $\phi=0.32$, in an extended range of wavenumbers including all essential features of $S(Q)$ and $H(Q)$. The x-ray scattering experiments were conducted in laminar flow to avoid radiation damage of the samples.

The recently developed analytic MPB-RMSA method for calculating $S(Q)$ of charged colloidal particles was used for a detailed analysis of the visible light- and x-ray scattering data, from which we have obtained quite accurate results for the colloidal volume fraction, $\phi$, and effective charge number $Z\left(\phi, n_{s}\right)$. Using the self-part corrected $\delta \gamma$-scheme with MPBRMSA input for $S(Q)$, we have obtained quite accurate results for the diffusion function $D(Q)=D_{0} H(Q) / S(Q)$, including the short-time collective and self-diffusion coefficients $D_{c}$ and $D_{s}$, and the cage diffusion coefficient $D_{\text {cge }}=D_{0} H\left(Q_{m}\right)$ as special cases. The results of the analytic theory are overall in very good agreement with the DLS/XPCS-determined hydrodynamic functions, for nominal volume fractions $\phi^{\text {nom }}$ $\leq 0.16$.

The analytic calculations are based on the purely repulsive, HSY-type pair potential consisting of the electrostatic part of the DLVO potential and an impermeable hard core. The static and short-time dynamics scattering data for the less polydisperse $\mathrm{PA}_{1117}$ samples with $\Delta R / R_{0} \approx 0.057$ are well described by the theoretically predicted ideal static structure factor $S(Q)$ and hydrodynamic function, $H(Q)$, of monodisperse HSY-like spheres. For the more polydisperse $\mathrm{PA}_{542}$ samples where $\Delta R / R_{0}=0.121$, the inclusion of incoherent scattering contributions within the decoupling approximation has significantly improved the quality of the structure factor fits, with practically quantitative agreement between the experimental curves and the decoupling approximation predictions for $S_{m}(Q) \approx S_{m}^{d}(Q)$ and $H_{m}(Q) \approx H_{m}^{d}(Q)$.

We find that the modified $\delta \gamma$-scheme becomes less accurate for the most concentrated samples (at $\phi^{\text {nom }} \approx 0.32$ ), at low salinities $n_{s}^{\text {nom }} \lesssim 5 \mathrm{mM}$. For these samples, no satisfactory analytical theoretical scheme has been developed so far that allows for an accurate prediction of $D_{s} / D_{0}$. At high salinities, however, Eq. (15) can be used for the selfdiffusion part of $H(Q)$ of the two $\mathrm{PA}_{1117}$ samples at the largest considered $\phi^{\text {nom }}$.

Our extensive experimental results have allowed us to study in detail the $\phi$ - and $n_{s}$-dependencies of the nearest neighbor cage radius $R_{m}$. In agreement with earlier theoretical predictions, we find $R_{m}$ to be proportional to $\phi^{-1 / 3}$ at very low values of $n_{s}$ only. For the low-salinity samples where this simple geometric scaling applies, the principal peak wavenumber, $Q_{m}$, is to good accuracy equal to $2.2 \pi / R_{m}$.

Also in agreement with theoretical predictions, our experimental findings for $D_{c} / D_{0}, D_{s} / D_{0}$, and $H\left(Q_{m}\right)$ are confined from above and below by the limiting concentration scaling forms for neutral hard spheres and strongly repelling HSYtype particles, respectively. The theoretically predicted hydrodynamical enhancement of cage-diffusion $\left(H\left(Q_{m}\right)>1\right)$ for 
low-concentrated systems at low salinity is confirmed by our experimental data.

\section{ACKNOWLEDGMENTS}

We thank Tina Autenrieth for help in the sample preparation and fruitful discussions, and Anders Madsen for support during the x-ray beamtime. This work was under appropriation of funds by the Deutsche Forschungsgemeinschaft (DFG), who supported F.W. within the Grant No. GRK 1355, and G.N. in Project No. B2 of SFB-TR6. M.H. acknowledges funding by the European Research Council (ERC) Advanced Grant INTERCOCOS, FP7 Ref. No. 267499.

\section{NOMENCLATURE}

DLS

DLVO

HIs

HS

HSY

MPB-

RMSA

PA

PA-scheme

SAXS

SLS

vdW

XPCS

Roman

$D(Q)$

$D_{m}(Q)$

$D_{c}$

$D_{\text {cge }}$

$D_{s}$

$D_{0}$

$g(r)$

$g_{1}(Q, \tau)$

$g_{2}(Q, \tau)$

$G(Q, \tau)$

$H(Q)$

$H_{d}(Q)$

$H_{m}(Q)$
Dynamic light scattering ${ }^{54}$

Derjaguin-Landau-Verwey-Overbeek (DLVO) pair potential $^{35}$

Hydrodynamic interactions ${ }^{11,14,57,58,60-64}$

Uncharged, non-overlapping hard spheres with hydrodynamic stick (i.e., no-slip) surfaces

Hard-sphere plus repulsive Yukawa (pair potential)

Modified penetrating background-corrected

rescaled mean spherical approximation ${ }^{9,10}$

Poly-acrylate

Approximation of pairwise additive HIs ${ }^{11,14,25}$

Small angle $x$-ray scattering ${ }^{29,30}$

Static light scattering ${ }^{54}$

van der Waals

X-ray photon correlation spectroscopy ${ }^{5,7}$

Ideal short-time diffusion function for a suspension of monodisperse spheres ${ }^{14,57}$

Measurable short-time diffusion function for a suspension of polydisperse spheres ${ }^{14}$

Collective diffusion coefficient, $D_{c}=D(Q$ $\rightarrow 0$ )

Cage diffusion coefficient, $D_{c g e}=D\left(Q_{m}\right)$

Self-diffusion coefficient, $D_{s}=D(Q \rightarrow \infty)$

Translational free diffusion coefficient of a single sphere with hydrodynamic stick (i.e., noslip) surface boundary conditions in an infinite quiescent fluid

Radial pair-distribution function ${ }^{55}$

Normalized field autocorrelation function ${ }^{54}$

Normalized intensity autocorrelation function $^{54}$

Self-intermediate scattering function ${ }^{55,56}$

Ideal hydrodynamic function for a suspension of monodisperse spheres ${ }^{14,57}$

Distinct part of the ideal hydrodynamic function, $H_{d}(Q)=H(q)-D_{s} / D_{0}$

Measurable hydrodynamic function for a suspension of polydisperse spheres ${ }^{14}$
$H_{m}^{d}(Q)$

$k$

$L_{B}$

$n_{s}$

$n_{s}^{\text {nom }}$

$\mathrm{PA}_{542}$

$\mathrm{PA}_{1117}$

$P_{m}(Q)$

$Q$

$Q_{m}$

$r$

$r_{m}$

$R_{m}$

$R_{0}$

$S(Q)$

$S_{m}(Q)$

$S_{m}^{d}(Q)$

$S(Q, \tau)$

$u(x)$

$W(\tau)$

$x$

$X(Q)$

Z

Greek

$\beta$

$\beta(Q)$

$\gamma$

$\delta \gamma$-scheme

$\Delta R / R_{0}$

$\epsilon$

$\tau$

$\tau_{B}$

$\tau_{H}$

$\tau_{I}$

$\phi$

$\phi^{\text {nom }}$
Measurable hydrodynamic function in decoupling approximation ${ }^{14}$

Yukawa screening parameter (inverse Debye screening length) $)^{35,47,48}$

Solvent-characteristic Bjerrum length

Number concentration of 1-1 electrolyte pairs in the solvent, includes added salt, water self-dissociation products, and adsorbed $\mathrm{CO}_{2}$, excludes counterions released from colloid surfaces

Experimentally selected (nominal) salt concentration

Batch of PA spherical particles with $R_{0}$ $=542 \AA$ and $\Delta R / R_{0}=0.121$

Batch of PA spherical particles with $R_{0}$ $=1117 \AA$ and $\Delta R / R_{0}=0.057$

Size-averaged particle form factor ${ }^{32}$

Scattering wavenumber, i.e., magnitude of scattering wavevector $\mathbf{Q}$

Scattering wavenumber at which the principal peaks in $S(Q)$ and $H(Q)$ occur

Colloidal center-to-center distance

Principal peak position of $g(r)$

Nearest neighbor shell radius, $R_{m}=r_{m}$

Mean hard-core radius of colloidal spherical particles

Ideal static structure factor for a suspension of monodisperse spheres ${ }^{55}$

Measurable static structure factor for a suspension of polydisperse spheres ${ }^{14}$

Measurable colloidal static structure factor in decoupling approximation ${ }^{14}$

Intermediate scattering function ${ }^{55}$

Pair potential of direct colloidal interactions in units of $x=r /\left(2 R_{0}\right)$

Colloidal mean squared displacement divided by factor $6^{14}$

Dimensionless separation of colloidal particle centers, $x=r /\left(2 R_{0}\right)$

Decoupling amplitude factor ${ }^{14}$

Colloidal effective charge number ${ }^{36-46}$

Inverse thermal energy. $\beta=1 /\left(k_{B} T\right)$

Speckle contrast ${ }^{54}$

Yukawa coupling parameter ${ }^{35}$

Renormalized concentration fluctuation expansion method of Beenakker and Mazur ${ }^{58-60}$

Relative standard deviation of the colloidal hard-core radius (polydispersity)

Zero-frequency solvent dielectric constant

Correlation time in time-resolved scattering experiments ${ }^{54}$

Colloidal momentum relaxation time $e^{14,57}$

Hydrodynamic vorticity diffusion time ${ }^{14,57}$

Colloidal interaction time ${ }^{14,57}$

Particle volume fraction

Experimentally selected (nominal) particle volume fraction 
${ }^{1}$ W. van Megen, R. H. Ottewill, S. M. Owens, and P. N. Pusey, J. Chem. Phys. 82, 508-515 (1985).

${ }^{2}$ P. N. Pusey and W. van Megen, Nature (London) 320, 340-342 (1986).

${ }^{3}$ S. B. Dierker, R. Pindak, R. M. Fleming, I. K. Robinson, and L. Berman, Phys. Rev. Lett. 75, 449-452 (1995).

${ }^{4}$ L. B. Lurio, D. Lumma, A. R. Sandy, M. A. Borthwick, P. Falus, S. G. J. Mochrie, J. F. Pelletier, M. Sutton, L. Regan, A. Malik, and G. B. Stephenson, Phys. Rev. Lett. 84, 785-788 (2000).

${ }^{5}$ G. Grübel, D. L. Abernathy, D. O. Riese, W. L. Vos, and G. H. Wegdam, J. Appl. Cryst. 33, 424-427 (2000).

${ }^{6}$ A. Malik, A. R. Sandy, L. B. Lurio, G. B. Stephenson, S. G. J. Mochrie, I. McNulty, and M. Sutton, Phys. Rev. Lett. 81, 5832-5835 (1998).

${ }^{7}$ D. L. Abernathy, G. Grübel, S. Brauer, I. McNulty, G. B. Stephenson, S. G. J. Mochrie, A. R. Sandy, N. Mulders, and M. Sutton, J. Synchrotron Radiat. 5, 37-47 (1998).

${ }^{8}$ G. Grübel and F. Zontone, J. Alloys Compd. 362, 3-11 (2004).

${ }^{9}$ M. Heinen, P. Holmqvist, A. J. Banchio, and G. Nägele, J. Chem. Phys. 134, 044532 (2011).

${ }^{10}$ M. Heinen, P. Holmqvist, A. J. Banchio, and G. Nägele, J. Chem. Phys. 134, 129901 (2011).

${ }^{11}$ M. Heinen, A. J. Banchio, and G. Nägele, J. Chem. Phys. 135, 154504 (2011).

${ }^{12}$ D. Chatenay, W. Urbach, R. Messager, and D. Langevin, J. Chem. Phys. 86, 2343-2351 (1987)

${ }^{13}$ B. D'Aguanno, U. Genz, and R. Klein, J. Phys.: Condens. Matter 2, SA379-SA384 (1990).

${ }^{14}$ G. Nägele, Phys. Rep. 272, 215-374 (1996).

${ }^{15}$ J. Gapinski, A. Wilk, A. Patkowski, W. Häußler, A. J. Banchio, R. Pecora, and G. Nägele, J. Chem. Phys. 123, 054708 (2005).

${ }^{16}$ M. Heinen, F. Zanini, F. Roosen-Runge, D. Fedunová, F. Zhang, M. Hennig, T. Seydel, R. Schweins, M. Sztucki, M. Antalík, F. Schreiber, and G. Nägele, Soft Matter 8, 1404-1419 (2012).

${ }^{17}$ A. P. Philipse and A. Vrij, J. Chem. Phys. 88, 6459-6470 (1988).

${ }^{18}$ J. K. Phalakornkul, A. P. Gast, R. Pecora, G. Nägele, A. Ferrante, B. MandlSteininger, and R. Klein, Phys. Rev. E 54, 661-675 (1996).

${ }^{19}$ W. Härtl, C. Beck, and R. Hempelmann, J. Chem. Phys. 110, 7070-7072 (1999).

${ }^{20}$ L. F. Rojas, R. Vavrin, C. Urban, J. Kohlbrecher, A. Stradner, F. Scheffold, and P. Schurtenberger, Faraday Discuss. 123, 385-400 (2003).

${ }^{21}$ A. J. Banchio, J. Gapinski, A. Patkowski, W. Häußler, A. Fluerasu, S. Sacanna, P. Holmqvist, G. Meier, M. P. Lettinga, and G. Nägele, Phys. Rev. Lett. 96, 138303 (2006).

${ }^{22}$ A. J. Banchio and G. Nägele, J. Chem. Phys. 128, 104903 (2008).

${ }^{23}$ J. Gapinski, A. Patkowski, A. J. Banchio, J. Buitenhuis, P. Holmqvist, M. P. Lettinga, G. Meier, and G. Nägele, J. Chem. Phys. 130, 084503 (2009).

${ }^{24}$ J. Gapinski, A. Patkowski, and G. Nägele, J. Chem. Phys. 132, 054510 (2010).

${ }^{25}$ M. Heinen, P. Holmqvist, A. J. Banchio, and G. Nägele, J. Appl. Crystallogr. 43, 970-980 (2010).

${ }^{26}$ S. C. Thickett and R. G. Gilbert, Polymer 48, 6965-6991 (2007).

${ }^{27}$ W. Härtl and X. Zhang-Heider, J. Colloid Interface Sci. 185, 398-401 (1997).

${ }^{28}$ G. Grübel, J. Als-Nielsen, and A. K. Freund, J. Phys. IV 4, 27-34 (1994).

${ }^{29}$ A. Guinier and G. Fournet, Small-Angle Scattering of X-rays (Wiley, New York, 1955)

${ }^{30}$ O. Glatter and O. Kratky, Small Angle X-ray Scattering (Academic, London, 1982)

${ }^{31}$ P. N. Pusey, H. M. Fijnaut, and A. Vrij, J. Chem. Phys. 77, 4270-4281 (1982).

${ }^{32}$ J. S. Pedersen, Adv. Colloid Interface Sci. 70, 171-210 (1997).

${ }^{33}$ G. V. Schulz, Z. Phys. Chem. B 43, 25-46 (1939).

${ }^{34}$ B. H. Zimm, J. Chem. Phys. 16, 1099-1116 (1948).

${ }^{35}$ E. J. W. Verwey and J. T. G. Overbeek, Theory of the Stability of Lyophobic Colloids (Elsevier, New York, 1948).
${ }^{36}$ L. Bocquet, E. Trizac, and M. Aubouy, J. Chem. Phys. 117(17), 8138-8152 (2002).

${ }^{37}$ E. Trizac, L. Bocquet, and M. Aubouy, Phys. Rev. Lett. 89(24), 248301 (2002).

${ }^{38}$ E. Trizac, L. Bocquet, M. Aubouy, and H. H. von Grünberg, Langmuir 19, 4027-4033 (2003).

${ }^{39}$ S. Pianegonda, E. Trizac, and Y. Levin, J. Chem. Phys. 126, 014702 (2007).

${ }^{40}$ A. Torres, G. Tellez, and R. van Roij, J. Chem. Phys. 128, 154906 (2008).

${ }^{41}$ M. G. McPhie and G. Nägele, Phys. Rev. E 78, 060401 (2008).

${ }^{42}$ T. E. Colla, Y. Levin, and E. Trizac, J. Chem. Phys. 131, 074115 (2009).

${ }^{43}$ S. Alexander, P. M. Chaikin, P. Grant, G. J. Morales, P. Pincus, and D. Hone, J. Chem. Phys. 80, 5776-5781 (1984).

${ }^{44}$ Y. Levin, M. C. Barbosa, and M. N. Tamashiro, Europhys. Lett. 41, 123127 (1998).

${ }^{45}$ M. N. Tamashiro, Y. Levin, and M. C. Barbosa, Physica A 258, 341-351 (1998).

${ }^{46}$ A. Diehl, M. C. Barbosa, and Y. Levin, Europhys. Lett. 53, 86-92 (2001).

${ }^{47}$ W. B. Russel and D. W. Benzing, J. Colloid Interface Sci. 83, 163-177 (1981).

${ }^{48}$ A. R. Denton, Phys. Rev. E 62, 3855-3864 (2000).

${ }^{49}$ I. K. Snook and J. B. Hayter, Langmuir 8, 2880-2884 (1992).

${ }^{50}$ J.-P. Hansen and J. B. Hayter, Mol. Phys. 46, 651-656 (1982).

${ }^{51}$ F. J. Rogers and D. A. Young, Phys. Rev. A 30, 999-1007 (1984).

${ }^{52}$ J. K. Percus and G. J. Yevick, Phys. Rev. 110, 1-13 (1958).

${ }^{53}$ M. S. Wertheim, Phys. Rev. Lett. 10, 321-323 (1963).

${ }^{54}$ B. J. Berne and R. Pecora, Dynamic Light Scattering: With Applications to Chemistry, Biology, and Physics (Wiley, New York, 1976).

${ }^{55}$ J.-P. Hansen and I. R. McDonald, Theory of Simple Liquids, 2nd ed. (Academic, London, 1986).

${ }^{56}$ R. Krause, G. Nägele, D. Karrer, J. Schneider, R. Klein, and R. Weber, Physica A 153(3), 400-419 (1988).

${ }^{57}$ J. K. G. Dhont, An Introduction to Dynamics of Colloids (Elsevier, Amsterdam, 1996).

${ }^{58}$ C. W. J. Beenakker and P. Mazur, Physica A 120, 388-410 (1983).

${ }^{59}$ C. W. J. Beenakker and P. Mazur, Physica A 126, 349-370 (1984).

${ }^{60}$ C. W. J. Beenakker, Physica A 128, 48-81 (1984).

${ }^{61}$ R. B. Jones and R. Schmitz, Physica A 149, 373-394 (1988).

${ }^{62}$ D. J. Jeffrey and Y. Onishi, J. Fluid Mech. 139, 261-290 (1984).

${ }^{63}$ S. Kim and S. J. Karrila, Microhydrodynamics: Principles and Selected Applications (Butterworth-Heinemann, Boston, 1991).

${ }^{64}$ G. C. Abade, B. Cichocki, M. L. Ekiel-Jeżewska, G. Nägele, and E. Wajnryb, J. Chem. Phys. 134, 244903 (2011).

${ }^{65}$ M. Heinen, P. Holmqvist, A. J. Banchio, and G. Nägele, "Short- and longtime dynamics of charged colloidal particles" (unpublished).

${ }^{66}$ A. Diehl and Y. Levin, J. Chem. Phys. 121, 12100-12103 (2004).

${ }^{67}$ Y. Zeng, S. Grandner, C. L. P. Oliveira, A. F. Thünemann, O. Paris, J. S. Pedersen, S. H. L. Klapp, and R. von Klitzing, Soft Matter 7, 10899-10909 (2011).

${ }^{68}$ S. H. L. Klapp, S. Grandner, Y. Zeng, and R. von Klitzing, J. Phys.: Condens Matter 20, 494232 (2008).

${ }^{69} \mathrm{~S}$. Busch, T. Jensen, Y. Chushkin, and A. Fluerasu, Eur. Phys. J. E 26, 5562 (2008).

${ }^{70}$ H. Löwen, T. Palberg, and R. Simon, Phys. Rev. Lett. 70, 1557-1560 (1993).

${ }^{71}$ M. Watzlawek and G. Nägele, Phys. Rev. E 56, 1258-1261 (1997).

${ }^{72}$ A. Robert, J. Wagner, W. Härtl, T. Autenrieth, and G. Grübel, Eur. Phys. J. E 25, 77-81 (2008).

${ }^{73}$ G. C. Abade, B. Cichocki, M. L. Ekiel-Jeżewska, G. Nägele, and E. Wajnryb, J. Chem. Phys. 132, 014503 (2010).

${ }^{74}$ J.-P. Hansen and L. Verlet, Phys. Rev. 184, 151-161 (1969).

${ }^{75}$ K. Kremer, M. O. Robbins, and G. S. Grest, Phys. Rev. Lett. 57, 2694-2697 (1986).

${ }^{76}$ M. J. Stevens and M. O. Robbins, J. Chem. Phys. 98, 2319-2324 (1993).

${ }^{77}$ D. C. Wang and A. P. Gast, J. Phys.: Condens Matter 11, 10133-10141 (1999). 